Article

\title{
Diaphorina citri Genome Possesses a Complete Melatonin Biosynthesis Pathway Differentially Expressed under the Influence of the Phytopathogenic Bacterium, Candidatus Liberibacter asiaticus
}

\author{
Yasser Nehela ${ }^{1,2}$ (D) and Nabil Killiny ${ }^{1, *(D)}$ \\ 1 Citrus Research and Education Center, Department of Plant Pathology, University of Florida, 700 Experiment \\ Station Rd., Lake Alfred, FL 33850, USA; yasser.nehela@ufl.edu \\ 2 Department of Agricultural Botany, Faculty of Agriculture, Tanta University, Tanta 31511, Egypt \\ * Correspondence: nabilkilliny@ufl.edu; Tel.: +1-863-956-8833
}

check for

updates

Citation: Nehela, Y.; Killiny, N. Diaphorina citri Genome Possesses a Complete Melatonin Biosynthesis Pathway Differentially Expressed under the Influence of the Phytopathogenic Bacterium, Candidatus Liberibacter asiaticus. Insects 2021, 12, 317. https://doi.org/ $10.3390 /$ insects 12040317

Academic Editor: Fabrice Legeai

Received: 10 March 2021

Accepted: 29 March 2021

Published: 1 April 2021

Publisher's Note: MDPI stays neutral with regard to jurisdictional claims in published maps and institutional affiliations.

Copyright: (c) 2021 by the authors. Licensee MDPI, Basel, Switzerland. This article is an open access article distributed under the terms and conditions of the Creative Commons Attribution (CC BY) license (https:// creativecommons.org/licenses/by/ $4.0 /)$.
Simple Summary: The indole-like compound, melatonin, is a tryptophan-derivative that is secreted by the pineal gland. Melatonin is ubiquitously distributed in both prokaryotes and eukaryotes including animals and plants. In animals, melatonin plays pleiotropic regulatory roles in several biological and physiological functions including sleep, circadian rhythm, oxidative stress, immune response, aging, apoptosis, and autophagy. Moreover, it might have anti-inflammatory, anti-tumor, and anti-cancer activities. Although most, if not all, of these genes were cloned and characterized previously in numerous animal species, none of them have been identified from the Asian citrus psyllid, Diaphorina citri, in the vector of Huanglongbing yet. In the current study, we performed a genome-wide analysis and introduces a shortlist of six putative melatonin biosynthesis-related genes included two putative tryptophan 5-hydroxylase (DcT5H-1 and DcT5H-2), a putative aromatic amino acid decarboxylase $(D c A A D C)$, two putative arylalkylamine $\mathrm{N}$-acetyltransferase (DcAANAT-1 and $D c A A N A T-2)$, and putative $\mathrm{N}$-acetylserotonin O-methyltransferase (DcASMT), which could indicate sites of functional or structural constraint. All these genes were differentially expressed under the influence of the phytopathogenic bacterium, Candidatus Liberibacter asiaticus, and after melatonin supplementation. Our findings could be a further step for optimization and cloning of melatonin biosynthesis genes of Diaphorina citri.

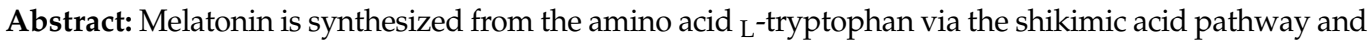
ubiquitously distributed in both prokaryotes and eukaryotes. Although most of melatonin biosynthesis genes were characterized in several plants and animal species including the insect model, Drosophila melanogaster, none of these enzymes have been identified from the Asian citrus psyllid, Diaphorina citri. We used comprehensive in silico analysis and gene expression techniques to identify the melatonin biosynthesis-related genes of $D$. citri and to evaluate the expression patterns of these genes within the adults of $D$. citri with gradient infection rates $(0,28,34,50,58$, and $70 \%)$ of the phytopathogenic bacterium Candidatus Liberibacter asiaticus and after the treatment with exogenous melatonin. We showed that the $D$. citri genome possesses six putative melatonin biosynthesis-related genes including two putative tryptophan 5-hydroxylase (DcT5H-1 and DcT5H-2), a putative aromatic amino acid decarboxylase $(D c A A D C)$, two putative arylalkylamine $\mathrm{N}$-acetyltransferase (DcAANAT-1 and DcAANAT-2), and putative $\mathrm{N}$-acetylserotonin O-methyltransferase (DcASMT). The infection with $\mathrm{Ca}$. $\mathrm{L}$. asiaticus decreased the transcript levels of all predicted genes in the adults of $D$. citri. Moreover, melatonin supplementation induced their expression levels in both healthy and Ca. L. asiaticus-infected psyllids. These findings confirm the association of these genes with the melatonin biosynthesis pathway.

Keywords: Diaphorina citri; Candidatus Liberibacter asiaticus; melatonin; citrus; tryptophan hydroxylase (TPH); aromatic L-amino acid decarboxylase (AADC); acetylserotonin methyltransferase (ASMT); serotonin $\mathrm{N}$-acetyltransferase (SNAT); bioinformatics 


\section{Introduction}

Huanglongbing (HLB; also known as citrus greening disease) is one of the most serious threatening diseases in citrus growing regions worldwide [1-4]. In the Americas, HLB was firstly confirmed in both South and North America (São Paulo, Brazil and Florida, USA, respectively) in 2004 and 2005, respectively [1-3]. Subsequently, it has been reported in Louisiana (2008), South Carolina and Georgia (2009), and Texas and California (2012) [3]. Additionally, it has been recorded in Caribbean countries included Belize, Cuba, Mexico, and Jamaica [3]. Recently, HLB turns out to be the most serious challenge for the citrus industry in Florida particularly, and the USA in general [5]. Diminishing the disease spread rate via reduction of vector populations has become one of the top priorities for the citrus industry, in California particularly, and in all citrus-growing areas generally [6-8].

HLB is associated with a fastidious, Gram-negative, phloem-limited, and non-culturable $\alpha$-proteobacterium Candidatus Liberibacter spp. [1-3]. Based on the characteristic $16 \mathrm{~S}$ rDNA sequence and geographical distribution, three Liberibacter species were proposed to be associated with HLB including $C a$. L. africanus in Africa, Ca. L. americanus in Brazil, and $\mathrm{Ca}$. L. asiaticus in Asia, Africa, and the Americas [1-3], with $\mathrm{Ca}$. L. asiaticus being the most dominant and destructive species worldwide [1,2]. Although Ca. Liberibacter spp. can be transmitted by graft inoculation, they are mainly transmitted by citrus psyllid vectors [9]. The African psyllid citrus, Trioza erytreae Del Guercio (Hemiptera: Triozidae) transmits the $C a$. L. africanus, while the Asian citrus psyllid, Diaphorina citri Kuwayama (Hemiptera: Liviidae), transmits both Ca. L. americanus and Ca. L. asiaticus [1].

The nature of $C a$. L. asiaticus $-D$. citri interactions extend from mutually beneficial to harmful [10]. For instance, infection with $C a$. L. asiaticus benefited $D$. citri by enhancing its reproductive fitness [10]. On the other hand, $\mathrm{Ca}$. L. asiaticus infection negatively affected the vector's susceptibility to insecticides [11], increased D. citri propensity for dispersal [12], exploited the energy metabolism and D. citri defense responses $[13,14]$, and shortened the lifespan and weakened the survival of the infected insects $[10,15]$. Interestingly, longevity (lifespan and survival) is strongly linked with numerous physiological issues, particularly the endogenous levels of melatonin. Our previous study showed that the reduced longevity of the infected psyllids was associated with lower endogenous melatonin content [15].

The indole-like compound, melatonin ( $N$-acetyl-5-methoxytryptamine), is a tryptophanderivative that is secreted by the pineal gland and ubiquitously distributed in various phylogenetically distant taxa including both prokaryotes and eukaryotes [15-18]. In animals, melatonin plays pleiotropic regulatory roles in several biological and physiological functions in vertebrates, invertebrates, and unicellular organisms. For example, it regulates sleep induction and circadian rhythm [19], oxidative stress [20], immune response and antiinflammatory activities [21], cell death and aging [22], and apoptosis and autophagy [23,24]. In addition, it might have potential anti-cancer [25,26] and anti-tumor [27] roles.

Melatonin is synthesized exclusively from the amino acid ${ }_{L}$-tryptophan via the shikimic

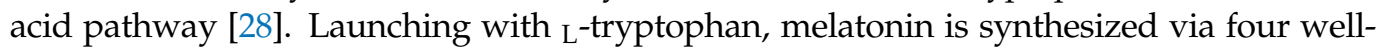
confirmed enzymatic steps in all living organisms [28,29]. In animals, $\mathrm{L}^{- \text {tryptophan is }}$ initially hydroxylated to 5-hydroxytryptophan using tryptophan hydroxylase (TPH; EC 1.14.16.4), then it is decarboxylated to form the key intermediate, serotonin (also known as 5-hydroxytryptamine), using aromatic amino acid decarboxylase (AADC; EC 4.1.1.28) [28]. Subsequently, serotonin is converted to melatonin via two consecutive enzymatic steps [29] using serotonin $\mathrm{N}$-acetyltransferase (SNAT; also known as arylalkylamine $\mathrm{N}$-acetyltransferase (AANAT); EC 2.3.1.87) and acetylserotonin O-methyltransferase (ASMT; formerly known as hydroxyindole-O-methyltransferase (HIOMT); EC 2.1.1.4) [28,30-32] via two potential pathways that are shared by both plants and animals.

In the first route, the penultima enzyme, SNAT, converts serotonin to $N$-acetylserotonin, while ASMT, the last enzyme, catalyzes $\mathrm{N}$-acetylserotonin to form the final product, melatonin $[28,30,32]$. Alternatively, serotonin could be methylated to 5-methoxytryptamine using ASMT, then $\mathrm{N}$-acetylated to produce melatonin using SNAT [31,33]. As mentioned above, both alternative routes for the biosynthesis of melatonin from serotonin are possibly 
occurring in both plants and animals and even microorganisms [28,29]. Nevertheless, distinct homologs of SNAT and ASMT have been reported between plants and animals, indicating their diverse origins during evolution [28,34].

Interestingly, most, if not all, of these genes were cloned and well-characterized previously in several plant species including Arabidopsis thaliana [31,35], Oryza sativa [32,36], Vitis vinifera [37,38], and the gymnosperm Pinus taeda [39]. Moreover, several melatonin biosynthesis-related genes have been cloned and characterized from numerous animal species including human [40,41], rats [42], guinea pig [43], chick [44], zebrafish [45], Atlantic croaker [46], and sheep [47]. Likewise, melatonin biosynthesis genes have been cloned and identified from the insect model, Drosophila melanogaster, including TPH (also known as tryptophan 5-hydroxylase (T5H)) [48,49], AADC (also known as dopa decarboxylase $(D D C)$ ) [50-52], and AANAT [53-55]. However, to the best of our knowledge, none of these enzymes have been previously identified from the Asian citrus psyllid, D. citri.

We believe that unguided functional genomic experiments are laborious and resourceintensive. The computational approaches can perform predictions with a variety of purposes which help to speed up the process in a more cost-efficient, and faster but more accurate manner. Our objectives for this study were to computationally identify the melatonin biosynthesis-related genes (TPH / T5H, AADC, SNAT / AANAT, and ASMT) in D. citri. Therefore, we employed comprehensive in silico and bioinformatics methods to (I) dig for melatonin biosynthesis-related genes homologies, (II) functionally analyze and predict active domains and important sites of these proteins, (III) model their three-dimensional structures, and (IV) investigate the ligand-receptor binding profile when possible. Furthermore, we evaluated the expression patterns of the predicted genes within the adults of $D$. citri after challenging with gradient infection rates of the phytopathogenic bacterium $\mathrm{Ca}$. L. asiaticus and after the treatment with exogenous melatonin. Our findings of this study are a further step for optimization and cloning of melatonin biosynthesis genes of $D$. citri.

\section{Materials and Methods}

2.1. In Silico Analysis

\subsubsection{Protein-Protein BLAST (BLASTp) Analysis}

The Basic Local Alignment Search tool (BLAST), particularly the Protein-Protein BLAST (BLASTp) algorithm, was used to dig and identify sequences of $D$. citri that resemble the query amino acid sequence of known melatonin biosynthesis-related enzymes of model insects. Briefly, the protein sequence of tryptophan hydroxylase (DmTPH, also known as DmT5H; GenBank Accession no. NP_612080.1) [48,49], dopa decarboxylase, isoform B (DmDDC, also known as DmAADC; GenBank Accession no. NP_724164.1) [50-52], arylalkylamine N-acetyltransferase 1, isoform A (DmAANAT1, also known as DmSNAT; GenBank Accession no. NP_523839.2) [53-55] from the fruit fly (Drosophila melanogaster), and $\mathrm{N}$-acetylserotonin O-methyltransferase-like protein, isoform X1 (ClASMT; GenBank Accession no. XP_014251646.1) from bed bug (Cimex lectularius) were matched with their homologous genes from D. citri using the Protein-Protein BLAST (BLASTP 2.8.0+) [56,57], based on recent available data on the two major databases included the "Diaphorina citri OGS v2.0 CDS" and "Diaphorina citri OGS v2.0 proteins" BLAST datasets available on the Citrus Greening Solutions website (https: / / citrusgreening.org/organism/Diaphorina_citri/ genome, 12 February 2021) [58] and the most recent available data in GenBank, the national center for biotechnology information website (NCBI, http: / / www.ncbi.nlm.nih.gov/gene/, 12 February 2021), using the compositionally adjusted substitution matrices [57]. Moreover, the Nucleotide-Nucleotide BLAST (BLASTn) algorithm was used to retrieve the nucleotide sequence for the top-matched sequences producing significant alignments with known melatonin-biosynthetic genes. Subsequently, a shortlist of top-matches was generated (Table 1) based on the phylogenetic trees, identity more than $50 \%$ (except for DcAANATs), and excluding all the hypothetical and low-quality proteins that have these characteristics. 
Table 1. Alignment statistics for the top-matched sequences producing significant alignments of melatonin-biosynthetic genes.

\begin{tabular}{|c|c|c|c|c|c|c|c|c|c|c|c|c|c|c|c|c|c|c|c|c|}
\hline \multirow[b]{3}{*}{$\begin{array}{c}\text { Gene } \\
\text { Description }\end{array}$} & \multirow[b]{3}{*}{ Gene ID } & \multirow{2}{*}{\multicolumn{6}{|c|}{$\begin{array}{c}\text { NCBI Database }{ }^{\mathrm{a}} \\
\text { Protein }\end{array}$}} & & & & & & \multicolumn{8}{|c|}{ D. citri Genome Database ${ }^{b}$} \\
\hline & & & & & & & & \multicolumn{5}{|c|}{ Protein-Protein Alignment Statistics } & \multicolumn{3}{|l|}{ Gene Description } & \multicolumn{2}{|l|}{ Protein } & \multicolumn{3}{|c|}{ Protein-Protein Alignment Statistics } \\
\hline & & Accession & bp & Accession & aа & $\begin{array}{l}\text { Theoretical } \\
\text { Isooelectric } \\
\text { Point (pI) }\end{array}$ & $\begin{array}{l}\text { Molecular } \\
\text { Weight } \\
\text { (MW) }\end{array}$ & $\begin{array}{l}\text { Max } \\
\text { Score }\end{array}$ & $\begin{array}{l}\text { Total } \\
\text { Score }\end{array}$ & $\begin{array}{l}\text { Query } \\
\text { Cover } \\
(\%)\end{array}$ & EValue & $\begin{array}{c}\text { Identity } \\
(\%)\end{array}$ & & Accession & bp & Accession & aа & $\begin{array}{c}\text { Identities } \\
(\%)\end{array}$ & $\begin{array}{c}\text { Positives } \\
(\%)\end{array}$ & E Value \\
\hline $\begin{array}{c}D c T 5 H^{c} \\
\text { Tryptophan } \\
\text { 5-hydroxylase } \\
\text { 1-like }\end{array}$ & $\begin{array}{c}\text { LOC } \\
113470334\end{array}$ & XM_0268828703.1 & 1343 & XP_026684504.1 & 379 & 5.64 & $43,291.92$ & 464 & 464 & 51 & $1 \times 10^{-161}$ & 75.69 & $\begin{array}{c}\text { DcT5H } \\
\text { Tryptophan } \\
\text { 5-hydroxylase, } \\
\text { putative }\end{array}$ & DeitrC076520.1.1 & 591 & DitrP076520.1.1 & 196 & 100 & 100 & $7 \times 10^{-143}$ \\
\hline $\begin{array}{c}\begin{array}{c}\text { Protein } \\
\text { henna-like }\end{array} \\
\text { hent }\end{array}$ & $\begin{array}{c}\text { LOC } \\
103524631\end{array}$ & ХM_017449691.2. & 1298 & XP_017305180.1 & 319 & 5.67 & $36,344.09$ & 363 & 363 & 58 & $5 \times 10^{-123}$ & 56.17 & Protein henna & DcitrC012845.1.1 & 1884 & DeitrP012845.1.1. & 627 & 87.15 & 87 & 0.0 \\
\hline$D C A A D C^{d}$ & & & & & & & & & & & & & $\begin{array}{c}\text { DCAADC (also } \\
\text { known as DCDDC) }\end{array}$ & & & & & & & \\
\hline $\begin{array}{c}\text { Aromatic-L- } \\
\text { amino-acid } \\
\text { decarboxylase } \\
\text { isoform X1 }\end{array}$ & $\begin{array}{c}\text { LOC } \\
103520978\end{array}$ & XM_008486080.3 & 1939 & XP_008484302.1 & 481 & 5.70 & $54,429.72$ & 734 & 734 & 90 & 0.0 & 75.27 & Dopa decarboxylase & DcitrC031955.1.1 & 1446 & DcitrP031955.1.1 & 481 & 100 & 100 & 0.0 \\
\hline $\begin{array}{l}\text { Aromatic-L- } \\
\text { amino-acid } \\
\text { decarboxylase }\end{array}$ & $\begin{array}{c}\text { LOC } \\
103510318\end{array}$ & XM_017444526.2 & 1737 & XP_017300015.1 & 484 & 6.61 & 54825.40 & 692 & 692 & 90 & 0.0 & 71.61 & Dopa decarboxylase & DeitrC031955.1.1 & 1446 & DcitrP031955.1.1 & 481 & 93.83 & 94 & 0.0 \\
\hline $\begin{array}{c}\text { DCAANAT (also } \\
\text { known as } \\
\text { DSSNAT) } \\
\text { e }\end{array}$ & & & & & & & & & & & & & $\begin{array}{c}\text { DcAANAT (also } \\
\text { known as DCSNAT) }\end{array}$ & & & & & & & \\
\hline $\begin{array}{l}\text { Dopamine N- } \\
\text { acetyltransferase- } \\
\text { like isform } \\
\text { X1 }\end{array}$ & $\begin{array}{c}\text { LOC } \\
103507708\end{array}$ & ХM_0268822511.1. & 1221 & XP_026678312.1. & 217 & 6.26 & $24,749.18$ & 67.4 & 67.4 & 85 & $1 \times 10^{-13}$ & 30.19 & $\begin{array}{c}\text { Dopamine } \\
\text { N-acetyltransferase }\end{array}$ & DcitrC025630.1.1 & 654 & DeitrP025630.1.1. & 217 & 99.08 & 99 & $8 \times 10^{-161}$ \\
\hline $\begin{array}{l}\text { Dopamine N- } \\
\text { acetyltransferase- } \\
\text { like }\end{array}$ & $\begin{array}{c}\text { LOC } \\
103507696\end{array}$ & XM_017443457.2 & 1911 & XP_017298946.1 & 220 & 5.39 & $24,808.39$ & 114 & 114 & 83 & $2 \times 10^{-31}$ & 34.78 & $\begin{array}{c}\text { Dopamine } \\
\text { N-acetyltransferase }\end{array}$ & DéitC0855745.1.1 & 723 & DeitrP085745.1.1 & 240 & 33.01 & 51.46 & $4 \times 10^{-30}$ \\
\hline $\begin{array}{l}D C A S M T \text { f } \\
\text { Septum } \\
\text { formation protein } \\
\text { Maf-like }\end{array}$ & $\begin{array}{c}\text { LOC } \\
113468045\end{array}$ & XM_026824671.1 & 746 & XP_026680472.1 & 162 & 5.26 & $18,064.58$ & 153 & 153 & 68 & $3 \times 10^{-47}$ & 50.00 & $\begin{array}{c}\text { DCASMT } \\
\text { N-actylserotonin } \\
\text { O-methyltransferase- } \\
\text { like }\end{array}$ & DcitrC032285.1.1 & 825 & DeitrP032285.1.1 & 274 & 98.77 & 100 & $5 \times 10^{-115}$ \\
\hline
\end{tabular}

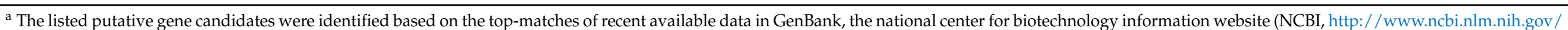

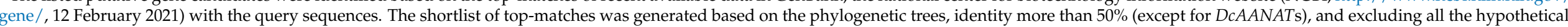

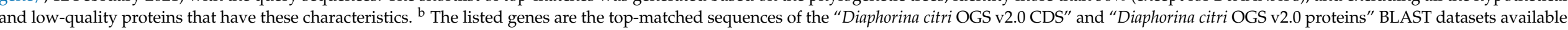

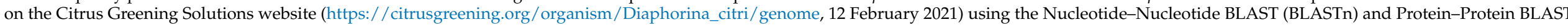

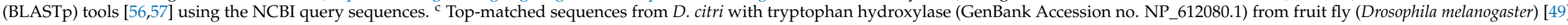

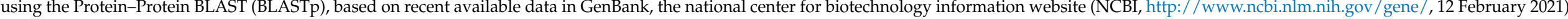

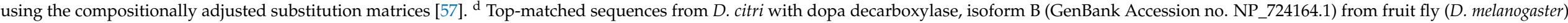

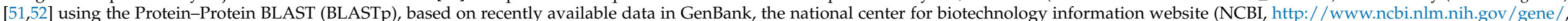

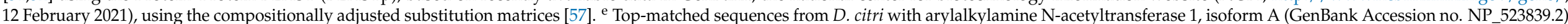

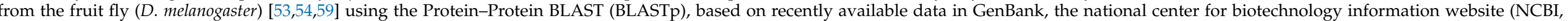

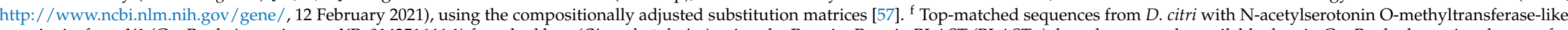

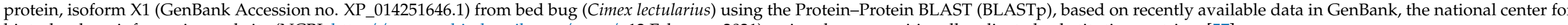
biotechnology information website (NCBI, http:/ / www.ncbi.nlm.nih.gov/gene/, 12 February 2021), using the compositionally adjusted substitution matrices [57]. 


\subsubsection{Evolutionary Analysis by Maximum Likelihood Method}

The evolutionary history of all matched sequences for each gene was inferred using the maximum likelihood method and JTT matrix-based model [60]. Initial tree(s) for the heuristic search were obtained automatically by applying Neighbor-Join and BioNJ algorithms to a matrix of pairwise distances estimated using the JTT model, and then selecting the topology with a superior log-likelihood value. Evolutionary analyses were conducted in MEGA X [61].

\subsubsection{Multiple Sequence Alignment Analysis}

Amino acid sequences from $D$. citri that produced significant alignments with known melatonin biosynthesis-related genes were simultaneously aligned using the ConstraintBased Alignment tool (COBALT; https://www.ncbi.nlm.nih.gov/tools/cobalt/re_cobalt. cgi, 12 February 2021) for multiple protein sequences [62]. Moreover, the top-matched sequences (amino acid and nucleotide sequences) of $D$. citri producing significant alignments with known melatonin-biosynthetic genes were used to generate the multiple sequence alignment by ClustalW (http:/ / www.genome.jp/tools-bin/clustalw, 12 February 2021) [63], and the version 3.21 of BOXSHADE (https:/ / embnet.vital-it.ch/software/BOX_form.html, 12 February 2021) was used to visualize conserved regions in the alignment.

\subsubsection{Conserved Domains and Theoretical $\mathrm{pI} / \mathrm{Mw}$}

The protein sequences were interactively classified into families and identified functionally important domains and conserved sites using The InterPro tool (https: / / www.ebi. ac.uk/interpro/, 12 February 2021) [64]. Moreover, the theoretical isoelectric point (pI) and molecular weight (MW) were computed using the Compute $\mathrm{pI} / \mathrm{Mw}$ tool (http:/ / web.expasy.org/compute_pi, 12 February 2021) [65].

\subsubsection{Three-Dimensional (3D) Structure Modeling and RNA Secondary Structure}

The SWISS-MODEL server (https: / / swissmodel.expasy.org/, 12 February 2021) [66], and the Protein Homology/Analogy Recognition Engine (Phyre2 Protein Fold Recognition Server, web portal-version 2.0; http://www.sbg.bio.ic.ac.uk/ phyre2/html/page.cgi?id= index, 12 February 2021) [67] were used for protein structure homology-modeling, structurebased function annotation, generating a three-dimensional (3D) structure, and prediction of membrane topology of the predicted sequences. The UCSF-Chimera package (version 1.15) (https: / / www.cgl.ucsf.edu/chimera/, 12 February 2021) [68] was used for interactive visualization of the predicted macromolecule (PDB format). Moreover, the RNAfold web server (http:/ / rna.tbi.univie.ac.at/cgi-bin/RNAWebSuite/RNAfold.cgi, 12 February 2021) [69] was used to predict RNA secondary structures using DNA sequences. To directly compare the folding stability of the predicted RNA secondary structures of various sizes, the minimum free energy (MFE) was obtained and the normalized MFE was calculated by dividing the MFE by the number of nucleotides (bp).

\subsection{Rearing of Healthy and Ca. L. asiaticus-Infected D. citri Colonies}

Healthy colonies of D. citri were continuously reared in a secured growth room $\left(27 \pm 2{ }^{\circ} \mathrm{C}, 60 \pm 5 \%\right.$ relative humidity, and $16: 8 \mathrm{~h} \mathrm{~L} / \mathrm{D}$ photocycle) at Citrus Research and Education Center (CREC), University of Florida $\left(28^{\circ} 10^{\prime} \mathrm{N}, 81^{\circ} 71^{\prime} \mathrm{E}\right)$, Lake Alfred, FL, USA. Insects were maintained on HLB-free alemow (Citrus macrophylla) trees. Random samples of $D$. citri adults and alemow leaves were collected monthly and tested for the presence of $C a$. L. asiaticus using polymerase chain reaction (PCR) assay as described by Tatineni et al. [70] to ensure that colonies remained HLB-free.

To obtain the $\mathrm{Ca}$. L. asiaticus-infected colonies, $D$. citri from the healthy colonies were reared on HLB-symptomatic and PCR-positive $\mathrm{C} a$. L. asiaticus-infected alemow trees and maintained in a separate secured growth room, under the same conditions as described above, to avoid cross-contamination. To obtain gradient infection rates of $D$. citri, the infection rates were tested monthly and prior to each experiment using two different 
methods: (I) Testing the presence of Ca. L. asiaticus visually in 50 individual psyllids using binocular laboratory compound microscope; and (II) the infection rates with Ca. L. asiaticus were further confirmed by PCR as described by Tatineni et al. [70]. Based on these examinations, $D$. citri colonies were categorized into five infection rates $(24,34,50,58$, and $70 \%)$, in addition to the healthy colony $(0 \%)$, which were all tested in this study. The infection rates were consistent without significant differences between them throughout the whole study. Briefly, newly emerged adults ( 2-days old) from each infection rate were collected using an aspirator without any sex-based discrimination.

\subsection{Treatment with Exogenous Melatonin}

Newly emerged D. citri adults (healthy versus Ca. L. asiaticus-infected (50\% infection rate)) were caged in the feeding system described in our previous study [15] and were fed on $100 \mu \mathrm{L}$ of $20 \%$ sucrose suspension as an artificial diet (mock control versus $500 \mu \mathrm{g} \mathrm{mL}^{-1}$ of melatonin in $20 \%$ sucrose solution). The artificial diet was placed between double-layered parafilm stretched over an acrylic feeding chamber that contained 50 adults (10 replicates/treatment). Insects were maintained at the same conditions as described above, and $72 \mathrm{~h}$ post-treatment (hpt), psyllids were collected using an aspirator for gene expression analysis.

\subsection{Gene Expression Analysis Using Quantitative Real-Time PCR (RT-PCR)}

Total RNA was extracted from five individual insects per replicate (10 replicates/treatment) using TriZol ${ }^{\circledR}$ reagent $\left(\right.$ Ambion ${ }^{\circledR}$, Life Technologies, New York, NY, USA), and the gene expression analysis was carried out as described in our previous study [15]. Quantification of transcript levels was used as a measure of the gene expression. Samples were analyzed in triplicate for each biological replicate. Primers for 6 melatonin biosynthesis-related genes (Table S1) were used to measure the gene expression. The relative expression of the consensus sequence among PCR products was determined according to the $2^{-\Delta \Delta C}$ T method [71]. For all gene expression experiments, data were normalized using two reference genes, $\alpha$-Tubulin and actin, which previously showed high stability for transcript normalization in D. citri under biotic stress [14].

\subsection{Statistical Analysis}

All experiments were designed in a completely randomized design using 10 biological replicates per treatment. The analysis of variance technique (ANOVA) was used for statistical comparison between more than two treatments, followed by post-hoc pairwise comparisons using the Tukey-Kramer honestly significant difference test (Tukey HSD; $p<0.05)$. Additionally, a two-tailed $t$-test was used for statistical comparison between only two treatments (healthy versus infected) or (mock-treated versus melatonin-treated), and statistical significance was established as $p<0.05$. Moreover, simple linear regression (SLR) analysis was performed to model the relationship between $\mathrm{C}$. . L. asiaticus infection rates (as an independent variable) and gene expression (as a dependent variable). The fitted regression line is expressed as a significant equation, as determined by the $\mathrm{F}$ test $(p<0.05)$. Both coefficients of determination $\left(\mathrm{R}^{2}\right)$ and adjusted coefficient of determination $\left(\mathrm{R}^{2}\right.$ adj $)$ were also obtained. Further, due to the observed nonlinear phenomena, data were fitted with a second-degree polynomial regression model (quadratic model) to understand the curvilinear relationship between $\mathrm{Ca}$. L. asiaticus infection rates (as an independent variable) and gene expression (as a dependent variable). Polynomial regression models, the $95 \%$ confident curves for the estimated regression, quadratic equation, $\mathrm{R}^{2}, \mathrm{R}^{2}$ adj, and $p$-value based on the F test $(p<0.05)$ were also obtained. JMP Statistical Software (SAS Institute, Cary, NC, USA) was used for all statistical analyses listed above. 


\section{Results}

\subsection{D. citri Genome Possesses a Putative Melatonin Biosynthetic Pathway}

The predicted melatonin biosynthesis pathway in D. citri was dissected using a comparative in silico analysis. Putative candidate genes involved in the melatonin biosynthesis pathway were presented as the top-matched sequences producing significant alignments of melatonin-biosynthetic genes from model insects and were selected based on sequence similarity, the phylogenetic relationships with the query sequences, and based on the sequence identity between query sequences and predicted ones, after excluding all the hypothetical and low-quality proteins that have these characteristics (Table 1).

\subsubsection{D. citri Genome Encodes for two Putative Tryptophan 5-hydroxylase (DcT5H)}

Using the Protein-Protein BLAST (BLASTp) tool, our findings showed that the D. citri genome possesses about six predicted amino acid sequences (based on NCBI database, Table S2) and nine sequences (based on Diaphorina citri OGS v2.0 proteins dataset, Table S3) that produce significant similarities to tryptophan hydroxylase $(\mathrm{DmT} 5 \mathrm{H}$, GenBank Accession no. NP_612080.1) from fruit fly (D. melanogaster). Although the multiple protein sequence alignment using COBALT analysis showed that all predicted sequences have relatively high homology with $\mathrm{DmT} 5 \mathrm{H}$ protein, the phylogenetic analysis showed that only two proteins from $D$. citri were phylogenetically closer to the query sequence (approximately $55 \%$ ) (Figure 1A). Those two proteins included putative tryptophan 5-hydroxylase 1-like (henceforth DcT5H-1) encoded by the D. citri locus LOC113470334 (GenBank Accession no. XP_026684504.1) and protein henna-like (henceforth DcT5H-2) by the D. citri locus LOC103524631 (GenBank Accession no. XP_017305180.1) (Figure 1A).

A
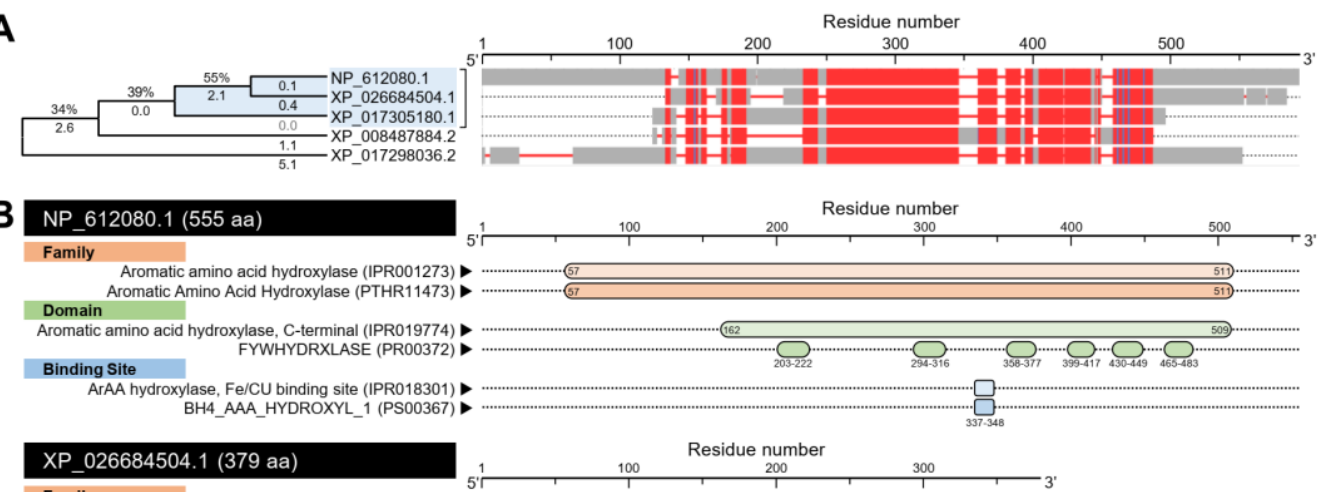

Family Aromatic amino acid hydroxylase (IPR001273)
Aromatic Amino Acid Hydroxylase (PTHR11473)
Domain

Domain
Aromatic amino acid hydroxylase, C-terminal (IPR019774)
FYWHYDRXXLAE (PR00372)

Binding Site FYWHYDRXLASE (PR00372)

ArAA hydroxylase, Fe/CU binding site (IPR018301)
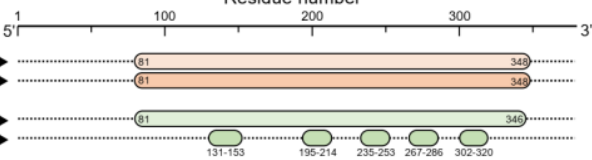

BH4_AAA_HYDROXYL_1 (PS00367)
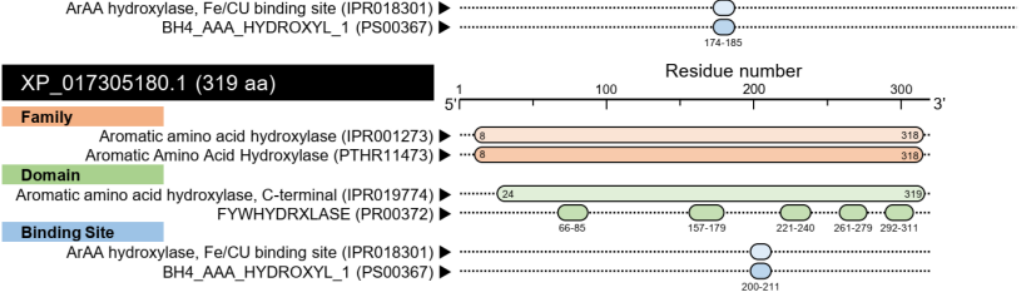

Figure 1. In silico analysis of tryptophan 5-hydroxylase (DcT5H) of Diaphorina citri. (A) Evolutionary analysis using maximum likelihood method and its associated multiple protein sequences alignments using Constraint-Based Alignment tool (COBALT) analysis. The AA sequences were identified using Protein-Protein BLAST (BLASTp) using tryptophan hydroxylase (DmT5H; GenBank Accession no. NP_612080.1) from Drosophila melanogaster, as a query sequence, against Diaphorina citri genome available in GenBank, the national center for biotechnology information website (NCBI, http: //www.ncbi.nlm.nih.gov/gene/, 12 February 2021). The tree with the highest log likelihood (-5340.89) is shown. The tree is drawn to scale, with branch lengths measured in the number of substitutions per site (next to the branches). The proportion of sites where at least one unambiguous base is present in at least 1 sequence for each descendent clade is shown next to each internal node in the tree. Evolutionary analyses and the joint tree were conducted in MEGA-X software. In the 
COBALT analysis, residues were colored using a column-based method according to their relative entropy threshold. Aligned columns with no gaps are colored blue and red, where the red color indicates highly conserved columns and blue indicates less conserved ones. (B) The protein functional and conserved domains analysis of DmT5H (NP_612080.1), DcT5H-1 (XP_026684504.1), and DcT5H-2 (XP_017305180.1) using the InterPro Scan tool (https://www.ebi.ac.uk/interpro/, 12 February 2021). FYWHYDRXLASE: Biopterin-dependent aromatic amino acid hydroxylase signature; ArAA_hydroxylase_Fe/CU: Aromatic amino acid hydroxylase, iron/copper-binding site; and BH4_AAA_HYDROXYL_1: Non-heme iron and tetrahydrobiopterin (BH4)-dependent enzymes.

The NCBI protein sequences of $D c T 5 H-1$ and $D c T 5 H-2$ were aligned with the sequence of the top-matched proteins from the D. citri database (DcitrP076520.1.1 and DcitrP012845.1.1, respectively). The AA alignment showed high similarity and conserved sequences in both proteins (Figures S1 and S2). Furthermore, the nucleotide sequence of DcT5H-1 (GenBank Accession no. XM_026828703.1) and DcT5H-2 (GenBank Accession no. XM_017449691.2) had high similarity and conserved sequences when aligned with the mRNA sequences from the D. citri database (DcitrC076520.1.1 and DcitrC012845.1.1, respectively) (Figures S3 and S4). Collectively, these findings suggest sequences retrieved from the NCBI database were highly similar and homology with those of the D. citri database. Therefore, we focused on these proteins for further in silico analysis.

The bioinformatic analysis of amino acid sequences using the InterPro Scan tool to interactively predict the conserved domains suggests a high topological similarity between DcT5Hs (DcT5H-1 and DcT5H-2) and DmT5H (Figure 1B). Briefly, all sequences have the tetrahydrobiopterin (BH4)-dependent aromatic amino acid (ArAA) hydroxylase family (IPR001273 and PTHR11473), and aromatic amino acid hydroxylase, C-terminal domain (IPR019774), FYWHYDRXLASE domain (PR00372), ArAA hydroxylase, Fe/CU binding site (IPR018301), and BH4_AAA_HYDROXYL_1 (PS00367) (Figure 1B).

The crystallographic three-dimensional (3D) structure of DmT5H, DcT5H-1, and DcT5H-2 was predicted using the crystal structure of human tryptophan 5-hydroxylase 2 (also known as tryptophan hydroxylase 2; TPH2), catalytic domain (Protein Data Bank (PDB ID): 4v06.1.A), and refined to $2.63 \AA$ resolution with excellent statistics (Figure 2).

Briefly, approximately $60 \%$ (residues Asp 174 to Ser 509) of DmT5H have been modeled with the template protein (seq identity $=67.16 \%$, seq similarity $=52 \%$, and confidence $=100 \%)$ with accepted global model quality estimation (GMQE $=0.40)$ and good absolute quality (QMEAN Z-score $=-1.80$ ) (Figure 2A). The predicted model of DmT5H is a monomer composed of $14 \alpha$-helices (three of them are short) and $14 \beta$-sheets (Figure 2A,B) with considerable predicted local similarity to target (Figure 2C). Further, it has two ligandbinding sites for iron (Fe; residues His 341, His 346, and Glu 386) and imidazole (IMD; residues Phe 310, Phe 319, Pro 337, His 341, Glu 342, His 346, and Glu 386) (Figure 2D).

Similarly, about 79\% (residues Asp 50 to Glu 349) of DcT5H-1 have been modeled with the target protein (seq identity $=60.54 \%$, seq similarity $=51 \%$, and confidence $=100 \%$ ) with high GMQE and QMEAN (0.60 and -2.20, respectively) (Figure 2E). The predicted model of DcT5H-1 contains $13 \alpha$-helix ribbons and 11 stranded $\beta$-wings (Figure 2E,F) with considerable predicted local similarity to the target (Figure 2G). Comparable to DmT5H, DcT5H-1 has two ligand-binding sites for iron (Fe; residues His 178, His 183, and Glu 223) and imidazole (IMD; seven residues included Phe 147, Phe 156, Pro 174, His 178, Glu 179, His 183, and Glu 223) (Figure 2H). Likewise, about 90\% (residues Phe 34 to Ile 319) of $\mathrm{DcT} 5 \mathrm{H}-2$ have been modeled with the target protein (seq identity $=70.28 \%$, seq similarity $=52 \%$, and confidence $=100 \%)$ with high GMQE $(0.75)$ and QMEAN $(-1.24)$ (Figure 2I). The predicted model of DcT5H-2 contains $14 \alpha$-helices (three were short) and 11 stranded $\beta$-sheets (Figure 2I,J) with considerable predicted local similarity to target (Figure 2K). Similar to DmT5H and DcT5H-1, DcT5H-2 had two ligand-binding sites for iron (Fe; residues His 204, His 209, and Glu 249) and imidazole (IMD; seven residues included Phe 173, Phe 182, Pro 200, His 204, Glu 205, His 209, and Glu 249) (Figure 2L). 


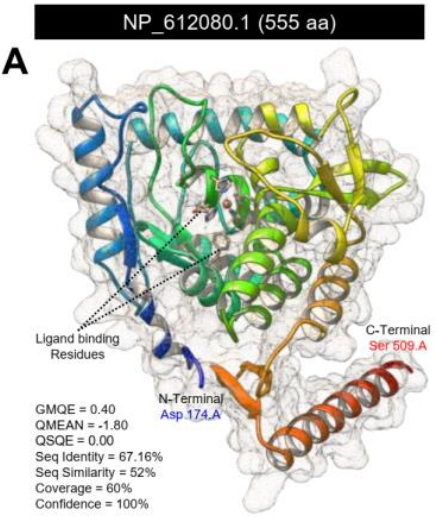

B
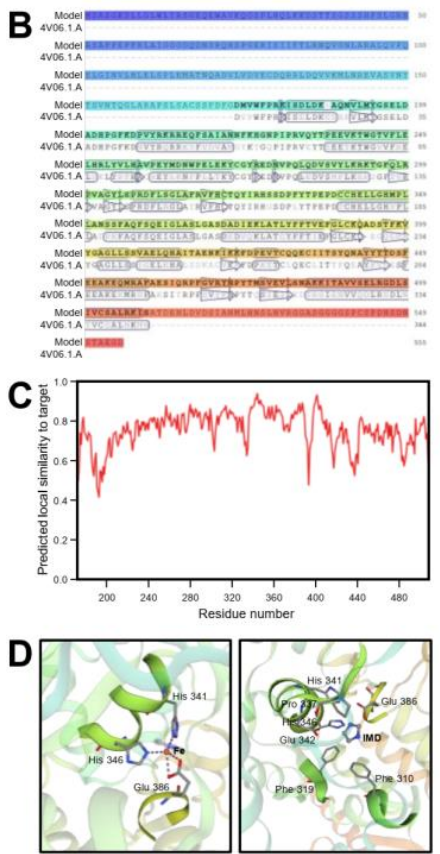

XP_026684504.1 (379 aa)

E

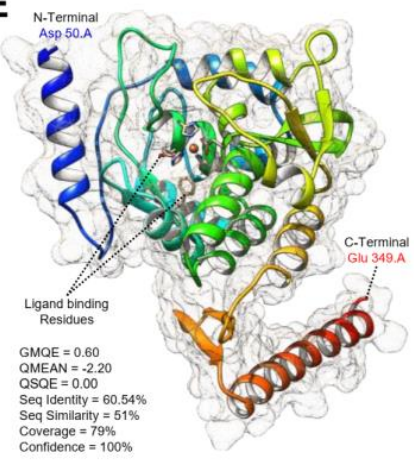

F
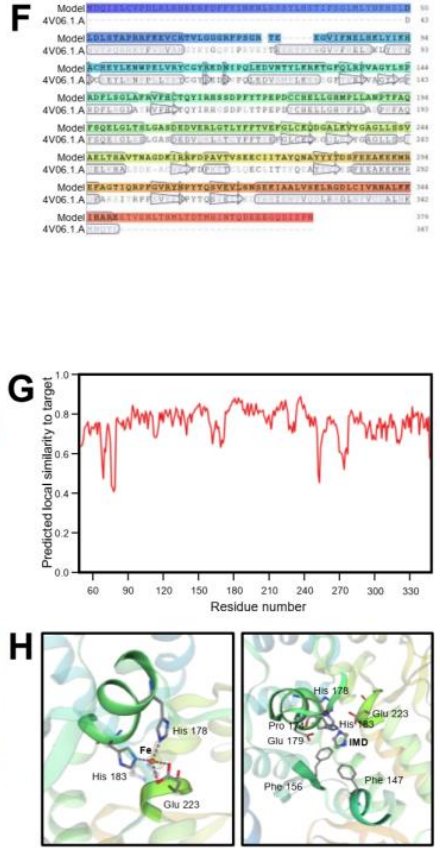
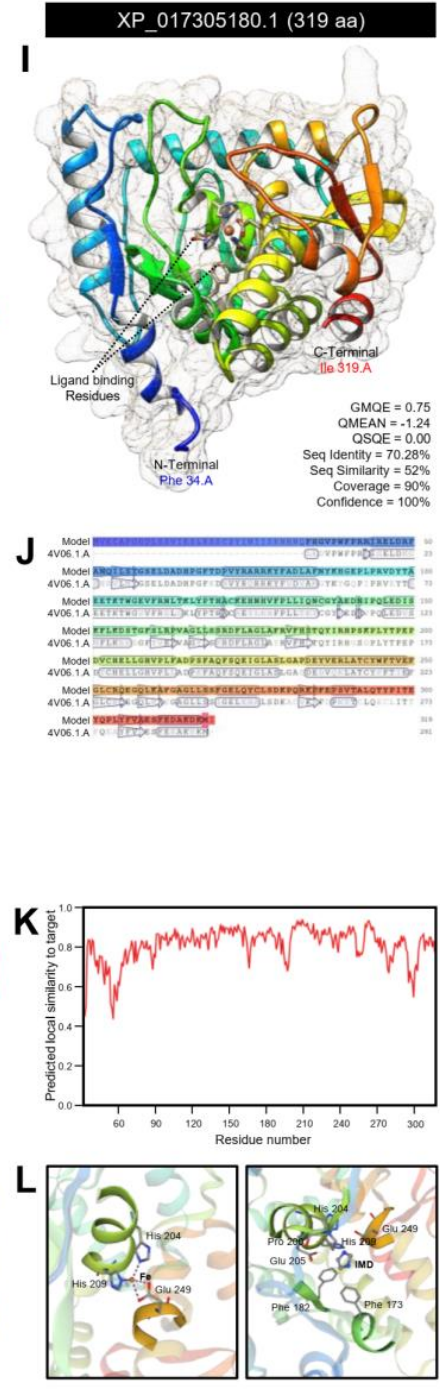

Figure 2. The crystallographic three-dimensional (3D) modeling of tryptophan 5-hydroxylase (DcT5H) of Diaphorina citri. (A,E,I) The predicted three-dimensional (3D) structure model and its associated mesh surface of DmT5H (NP_612080.1), DcT5H-1 (XP_026684504.1), and DcT5H-2 (XP_017305180.1), respectively. The tertiary structures were predicted with $100.0 \%$ confidence by the single highest scoring template of the crystal structure of human tryptophan 5-hydroxylase 2 (also known as tryptophan hydroxylase 2; TPH2), catalytic domain (Protein Data Bank (PDB ID): 4v06.1.A), and refined to $2.63 \AA$ resolution. Protein chains are colored according to the rainbow color spectrum, from blue (N-terminus) to red (C-terminus). (B,F,J) Model-template alignment of DmT5H, DcT5H-1, and DcT5H-2, respectively. AA sequences of each model were aligned with the template (4v06.1.A). Secondary structures are represented by rectangles ( $\alpha$-helices) and arrows ( $\beta$-sheets). Matched sequences are indicated in black. $(\mathbf{C}, \mathbf{G}, \mathbf{K})$ Local quality estimate of the predicted models of $\mathrm{DmT} 5 \mathrm{H}, \mathrm{DcT} 5 \mathrm{H}-1$, and DcT5H-2, respectively. $(\mathbf{D}, \mathbf{H}, \mathbf{L})$ Close-up of the ligand-binding site of $\mathrm{DmT} 5 \mathrm{H}, \mathrm{DcT} 5 \mathrm{H}-1$, and $\mathrm{DcT} 5 \mathrm{H}-2$, respectively. The selected poses were oriented to show the entry point for iron (Fe) and imidazole (IMD). Some surrounding AA near to the ligand are shown with their residue number. All bioinformatics analyses were carried out based on recent available data on the "Diaphorina citri OGS v2.0 proteins" dataset available on Citrus Greening Solutions website (https:/ / citrusgreening.org/organism/Diaphorina_citri/genome, 12 February 2021) and the most recent available data in GenBank, the national center for biotechnology information website (NCBI, 
http:/ /www.ncbi.nlm.nih.gov/gene/, 12 February 2021). The 3D structure was created using the SWISS-MODEL server (https:/ / swissmodel.expasy.org/, 12 February 2021) and visualized with the UCSF-Chimera package (version 1.15) (https:/ / www.cgl.ucsf.edu/chimera/, 12 February 2021). GMQE: Global model quality estimation and QSQE: Quaternary structure quality estimate.

Moreover, using the nucleotide sequence, we predicted the mRNA hairpins of DmT5H (NM_138236.2; 2183 bp), DcT5H-1 (XM_026828703.1; 1343 bp), and DcT5H-2 (XM_017449691.2; $1298 \mathrm{bp}$ ) with strengths of base pairing probabilities of minimum free energy (MFE; Figure $3 \mathrm{~A}-\mathrm{C}$, respectively) and centroid secondary structures (Figure 3D-F, respectively). The results obtained with RNAfold analysis demonstrated that the mRNA hairpins of DmT5H, DcT5H-1, and DcT5H-2 could generate a stable MFE secondary structure (MFE $=-753.40$, -356.10 , and $-377.80 \mathrm{Kcal} / \mathrm{mol}$, respectively) and centroid secondary structure (MFE $=-553.52$, -288.90 , and $-228.90 \mathrm{Kcal} / \mathrm{mol}$, respectively). Furthermore, normalized MFE has been used for a direct comparison of the folding stability of predicted mRNA hairpins of various sizes. The MFE secondary structure of $\mathrm{DcT} 5 \mathrm{H}-1$ was more stable (normalized MFE $=-0.2652$; Figure 3B) than DcT5H-2 (normalized MFE $=-0.2911$; Figure 3C) and DmT5H (normalized MFE $=-0.3451$; Figure 3A). Whereas the centroid secondary structure of DcT5H-2 was more stable (normalized $\mathrm{MFE}=-0.1763$; Figure 3F) than DcT5H-1 (normalized MFE $=-0.2151$; Figure 3E) and DmT5H (normalized MFE $=-0.2536$; Figure 3D).
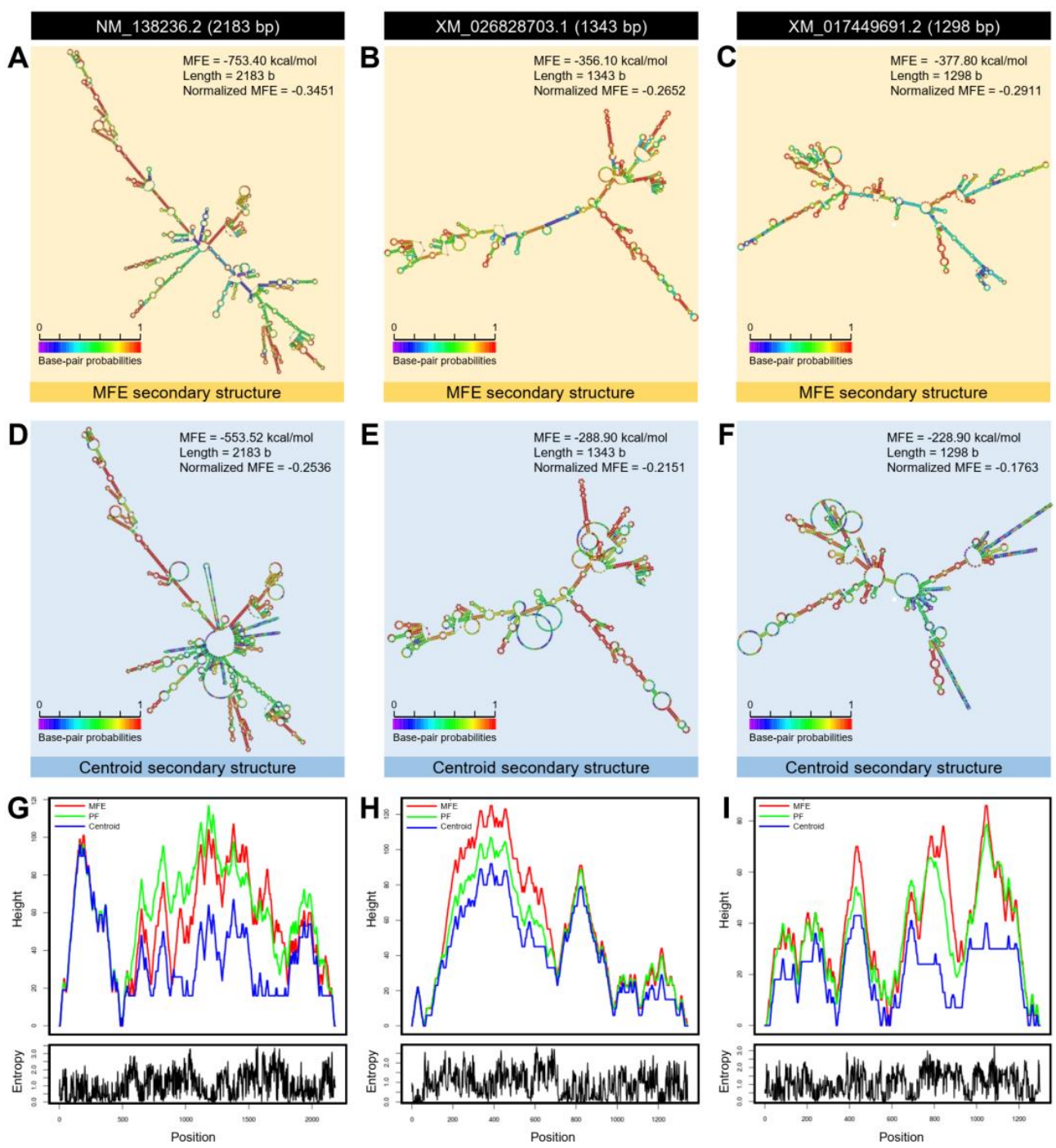

Figure 3. mRNA hairpins of tryptophan 5-hydroxylase (DcT5H) of Diaphorina citri. (A-C) Predicted minimum free energy (MFE) secondary structure of DmT5H (NM_138236.2), DcT5H-1 (XM_026828703.1), 
and DcT5H-2 (XM_017449691.2), respectively. (D-F) Predicted centroid secondary structure of DmT5H, DcT5H-1, and DcT5H-2, respectively. Colors represent strengths with base-pairing probabilities. (G-I) The mountain plot representations of the MFE structure, the centroid structure, the thermodynamic ensemble of mRNA structures, and the positional entropy of DmT5H, DcT5H-1, and $\mathrm{DcT} 5 \mathrm{H}-2$, respectively. mRNA secondary structures were predicted using RNAfold web server (http:/ / rna.tbi.univie.ac.at/cgi-bin/RNAWebSuite/RNAfold.cgi, 12 February 2021) using the nucleotide sequences.

Additionally, the mountain plot representation of the MFE structure, the centroid structure, the thermodynamic ensemble of RNA structures, and the positional entropy of DmT5H (Figure 3G), DcT5H-1 (Figure 3H), and DcT5H-2 (Figure 3I) suggested that all predicted secondary RNA structures were thermodynamically stable with the superiority of DcT5H-1. Further, no significant disparity was observed among the predicted structures which can be considered as proof of the stability of the secondary structures.

\subsubsection{D. citri Genome Encodes for a Putative Aromatic Amino Acid Decarboxylase (DcAADC)}

In silico analysis using the BLASTp tool showed that the D. citri genome possesses about 15 sequences (based on NCBI database, Table S4) and 16 sequences (based on Diaphorina citri OGS v2.0 proteins dataset, Table S5) that produce significant similarities to dopa decarboxylase, isoform B (DmDDC, also known as aromatic L-amino acid decarboxylase (DmAADC); GenBank Accession no. NP_724164.1) from D. melanogaster. The multiple protein sequence alignment using COBALT analysis using the NCBI sequences showed that all predicted sequences have relatively high homology with DmDDC protein (Figure 4A). The phylogenetic analysis showed that only three proteins from $D$. citri were phylogenetically closer to the query sequence (Figure $4 \mathrm{~A}$ ). These proteins included aromatic L-amino acid decarboxylase, isoform X1 (henceforth DcAADC-1) encoded by the D. citri locus LOC103520978 (481aa; GenBank Accession no. XP_008484302.1), aromatic L-amino acid decarboxylase (henceforth $D c A A D C-2$ ) encoded by the $D$. citri locus LOC103510318 (484; GenBank Accession no. XP_017300015.1), and aromatic L-amino acid decarboxylase-like encoded by the D. citri locus LOC103510317 (93 aa; GenBank Accession no. XP_026680193.1) (Figure 4A). However, the latest sequence was excluded from our further analysis because it was very short compared with the query sequence, and it had low query cover (14\%; Table S4).

Interestingly, both protein sequences (DcAADC-1 and $D c A A D C-2)$ were highly similar to each other and showed conserved sequences to the top-matched protein from the D. citri database (DcitrP031955.1.1; 481 aa) (Figure S5). Likewise, the nucleotide sequence of DcAADC-1 (1939 bp, GenBank Accession no. XM_008486080.3) and DcAADC-2 (1737 bp, GenBank Accession no. XM_017444526.2) had high similarity and conserved sequences when aligned with the mRNA sequences from the D. citri database (1446 bp, DcitrC031955.1.1) (Figure S6). Together, these findings suggest both sequences retrieved from the NCBI database presented the same protein sequence from the $D$. citri database.

The prediction of the conserved domains using the InterPro Scan tool suggests a high topological similarity among $D m A A D C, D c A A D C-1$, and $D c A A D C-2$ (Figure 4B). All sequences had two families included aromatic L-amino acid decarboxylase (IPR010977) and pyridoxal phosphate-dependent decarboxylase (IPR002129); three homologous pyridoxal phosphate-dependent transferase superfamilies (IPR015424, IPR015421, and IPR015422); and two binding sites including the pyridoxal-phosphate binding site (IPR021115) and DDC/GAD/HDC/TyrDC pyridoxal-phosphate attachment site (PS00392) (Figure 4B). 


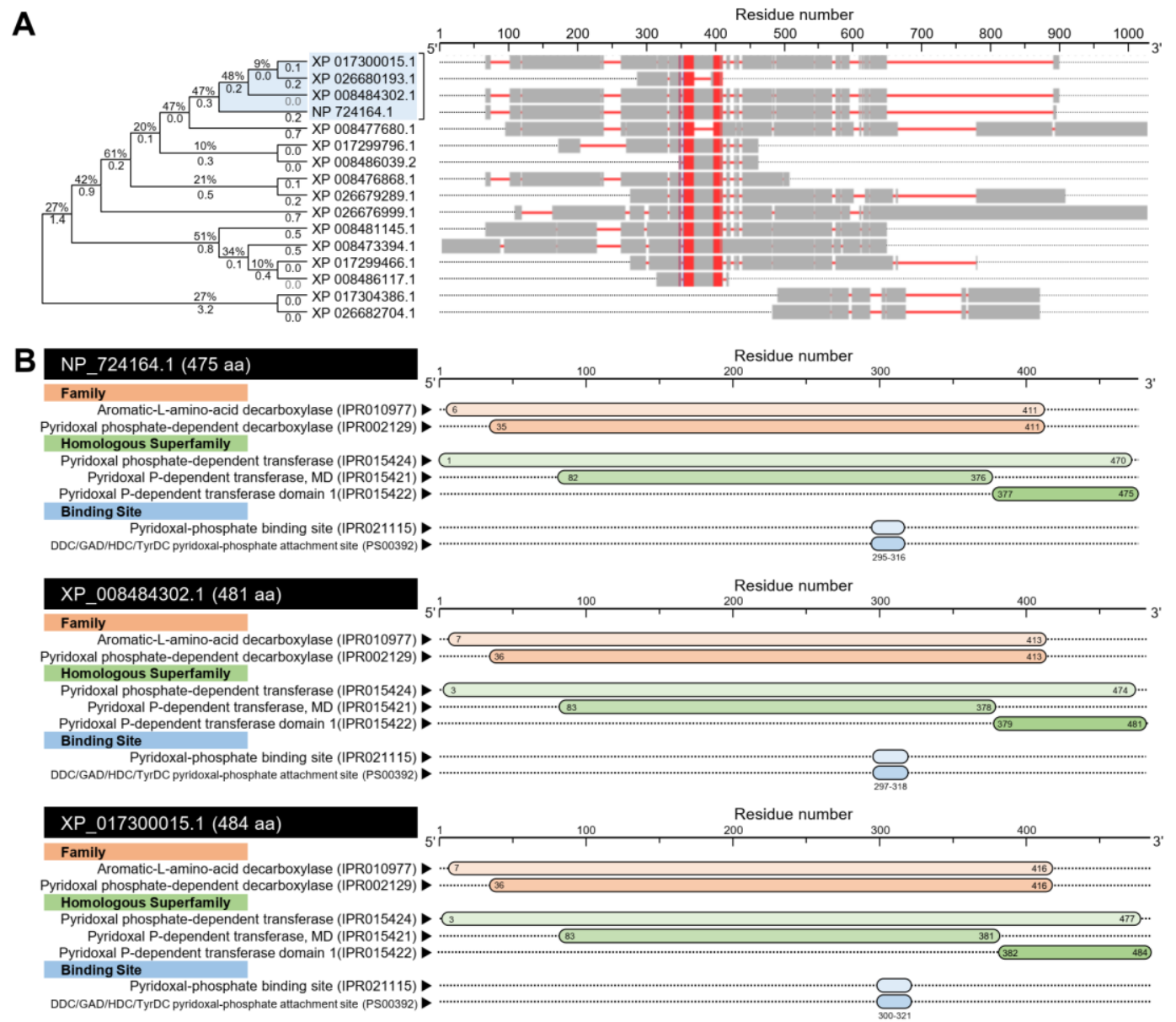

Figure 4. In silico analysis of aromatic L-amino acid decarboxylase (DcAADC) of Diaphorina citri. (A) Evolutionary analysis using maximum likelihood method and its associated multiple protein sequences alignments using ConstraintBased Alignment tool (COBALT) analysis. The AA sequences were identified using the Protein-Protein BLAST (BLASTp) using dopa decarboxylase, isoform B (GenBank Accession no. NP_724164.1) from Drosophila melanogaster, as a query sequence, against Diaphorina citri genome available in GenBank, the national center for biotechnology information website (NCBI, http:/ / www.ncbi.nlm.nih.gov/gene/, 12 February 2021). The tree with the highest log likelihood (-11,911.42) is shown. The tree is drawn to scale, with branch lengths measured in the number of substitutions per site (next to the branches). The proportion of sites where at least one unambiguous base is present in at least one sequence for each descendent clade is shown next to each internal node in the tree. Evolutionary analyses and the joint tree were conducted in MEGA-X software. In the COBALT analysis, residues were colored using a column-based method according to their relative entropy threshold. Aligned columns with no gaps are colored blue and red, where the red color indicates highly conserved columns and blue indicates less conserved ones. (B) The protein functional and conserved domains analysis of DmAADC (NP_724164.1), DcAADC-1(XP_008484302.1), and DcAADC-2 (XP_017300015.1) using InterPro Scan tool (https://www.ebi.ac.uk/interpro/, 12 February 2021). Pyridoxal P-dependent transferase, MD: Pyridoxal phosphate-dependent transferase, major domain; DDC: Aromatic L-amino acid decarboxylase; GAD: Glutamate decarboxylase; HDC: Histidine decarboxylase; and TyrDC: Tyrosine decarboxylase.

The crystallographic 3D structure of $D m A A D C, D c A A D C-1$, and $D c A A D C-2$ was predicted using the crystal structure of Drosophila 3,4-dihydroxyphenylalanine decarboxylase (also known as aromatic L-amino acid decarboxylase; PDB ID 3k40.1.A) and refined to 1.75 Å resolution with excellent statistics (Figure 5). DmAADC was predicted as a homodimer composed of two identical units. Each unit represented a $100 \%$ coverage (residues Met 1 to Gln 475) with the template protein with remarkable statistics (GMQE $=0.95$, QMEAN $=-0.80$, QSQE $=0.92$, seq identity $=100 \%$, seq similarity $=62 \%$, and confidence $=100 \%$ ) (Figure 5A). Further, each unit was composed of $23 \alpha$-helices, 11 long 
$\beta$-sheets, and six short $\beta$-sheets (Figure $5 \mathrm{~A}, \mathrm{~B}$ ) with high predicted local similarity to target (Figure 5C).
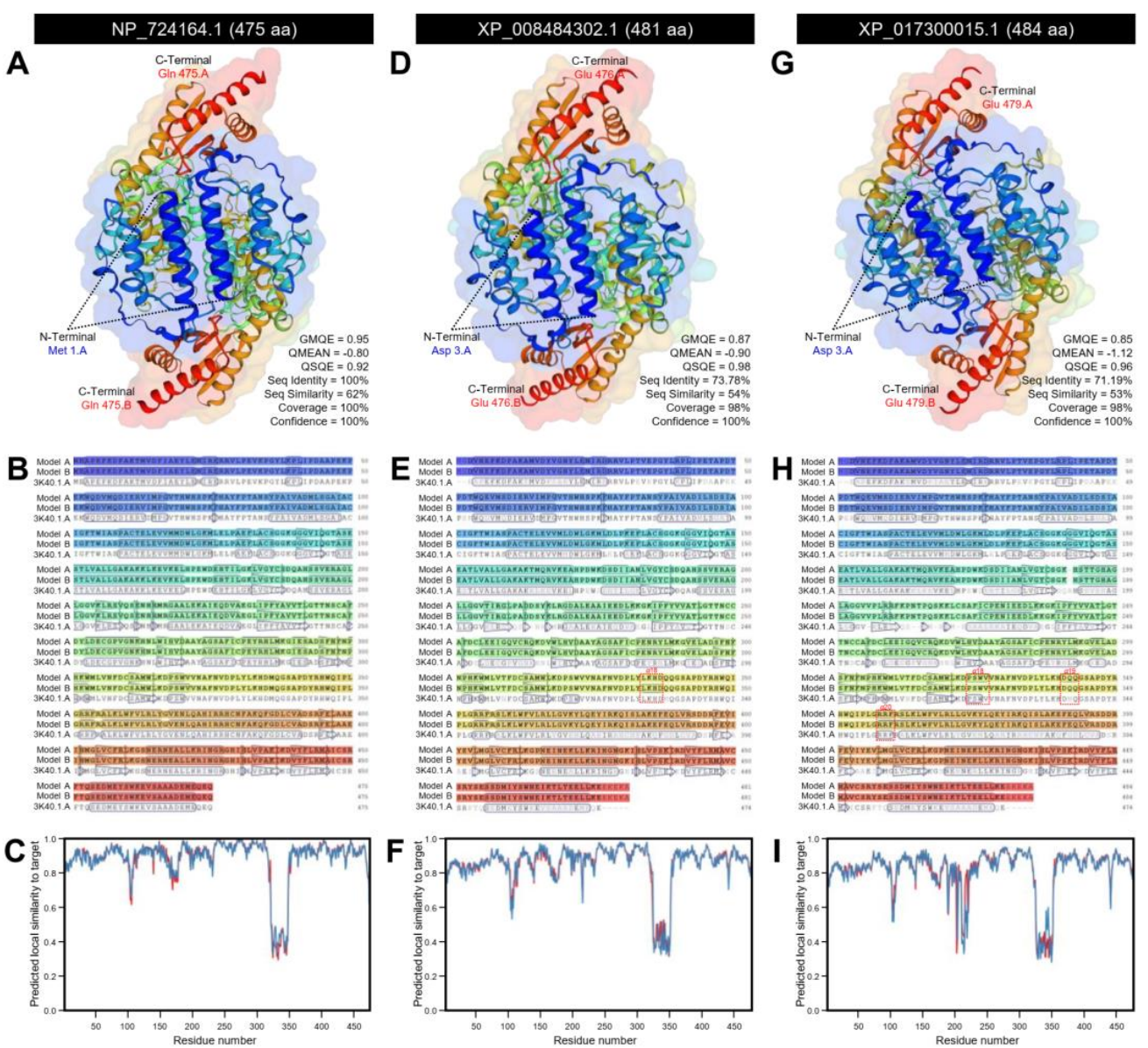

Figure 5. The crystallographic three-dimensional (3D) modeling of aromatic L-amino acid decarboxylase (DcAADC) of Diaphorina citri. $(\mathrm{A}, \mathrm{D}, \mathrm{G})$ The predicted three-dimensional (3D) structure model and its associated surface of DmAADC (NP_724164.1), DcAADC-1(XP_008484302.1), and DCAADC-2 (XP_017300015.1), respectively. The tertiary structures were predicted with $100.0 \%$ confidence by the single highest scoring template of the crystal structure of Drosophila 3,4dihydroxyphenylalanine decarboxylase (also known as aromatic L-amino acid decarboxylase; PDB ID 3k40.1.A) and refined to $1.75 \AA$ resolution. Protein chains are colored according to the rainbow color spectrum, from blue (N-terminus) to red (C-terminus). (B,E,H) Model-template alignment of $D m A A D C, D c A A D C-1$, and $D c A A D C-2$, respectively. AA sequences of each model were aligned with the template ( $3 \mathrm{k} 40.1$.A). Secondary structures are represented by rectangles ( $\alpha$-helices) and arrows ( $\beta$-sheets). Matched sequences are indicated in black. (C,F,I) Local quality estimate of the predicted models of $D m A A D C, D c A A D C-1$, and $D c A A D C-2$, respectively. All bioinformatics analyses were carried out based on recent available data on the "Diaphorina citri OGS v2.0 proteins" dataset available on Citrus Greening Solutions website (https:/ / citrusgreening.org/organism/Diaphorina_citri/genome, 12 February 2021) and the most recent available data in GenBank, the national center for biotechnology information website (NCBI, http:/ / www.ncbi.nlm.nih.gov/gene/, 12 February 2021). The 3D structure was created using the SWISS-MODEL server (https: / / swissmodel.expasy.org/, 12 February 2021) and visualized with the UCSF-Chimera package (version 1.15) (https: / www.cgl.ucsf.edu/chimera/, 12 February 2021). GMQE: Global model quality estimation and QSQE: Quaternary structure quality estimate.

Likewise, $D c A A D C-1$ and $D c A A D C-2$ were also predicted as homodimers that combined two units (model A and model B) with slight differences between both units in each model (Figure 5). Briefly, the predicted model of DcAADC-1 covered $98 \%$ with the target 
protein (residues Asp 3 to Glu 476) with notable statistics (GMQE = 0.87, QMEAN $=-0.90$, QSQE $=0.98$, seq identity $=73.78 \%$, seq similarity $=54 \%$, and confidence $=100 \%$ ) and were composed of $21 \alpha$-helix ribbons for model A or $22 \alpha$-helices for model B and 17 stranded $\beta$-sheets for each model (six of them were short) (Figure $5 \mathrm{D}, \mathrm{E}$, respectively) with considerable predicted local similarity to target (Figure 5F). The extra $\alpha$-helix of model B ( $\alpha 18)$ was represented by four residues including Leu 334, Lys 335, His 336, and Asp 337 (Figure 5E).

Similarly, in each unit of DCAADC-2, approximately $98 \%$ of the protein sequence (residues Asp 3 to Glu 479) have been modeled with the target protein with significant statistics $(\mathrm{GMQE}=0.85, \mathrm{QMEAN}=-1.12$, QSQE $=0.96$, seq identity $=71.19 \%$, seq similarity $=53 \%$, and confidence $=100 \%$ ) (Figure 5G). Model A of DcAADC-2 had $23 \alpha$-helix ribbons, whereas model B had only $20 \alpha$-helices, and both models had $15 \beta$-sheets each (four of them were short) (Figure $5 \mathrm{G}, \mathrm{H}$ ) with high predicted local similarity to target (Figure 5I). Model A of DcAADC-2 had three extra $\alpha$-helix ribbons $(\alpha 18, \alpha 19$, and $\alpha 20$, respectively). The ribbon $\alpha 18$ was composed of four residues (Pro 323, Ser 324, Trp 325, and Val 326), $\alpha 19$ composed of three residues (Asp 340, Gln 341, and Gln 342), and $\alpha 20$ composed of three residues also (Arg 357, Arg 358, and Phe 359) (Figure 5H).

Furthermore, the structure and/or function of the three protein sequences (DmAADC, $D c A A D C-1$, and $D c A A D C-2)$ were deeply analyzed using the Phyre2 protein fold recognition server (Figure 6). The crystallographic 3D structures of the three proteins were predicted using the same protein template (PDB ID 3k40.1.A). All 3D structures were predicted as monomers and they were almost identical (Figure 6A-C) except for only one $\alpha$-helix ribbon that was present in $D m A A D C, D c A A D C-1$ (Figure 6D,E, respectively), but it was absent in $D c A A D C$-2 (Figure 6F). Additionally, Phyre2-based predicted topology suggested that the three predicted proteins (DmAADC, DcAADC-1, and DcAADC-2) might act as transporters. The predicted topology of $D m A A D C$ showed that it contains four transmembrane domains (S1-S4) (three connecting loops, and internal N- and C-termini (Figure 6G)) DcAADC-1 contains five transmembrane domains (S1-S5) (four connecting loops, internal N-terminal, and external C-terminal (Figure $6 \mathrm{H})$ ), and DcAADC-2 contains six transmembrane domains (S1-S6) (five connecting loops, and internal N- and C-terminal extremities (Figure 6I)).
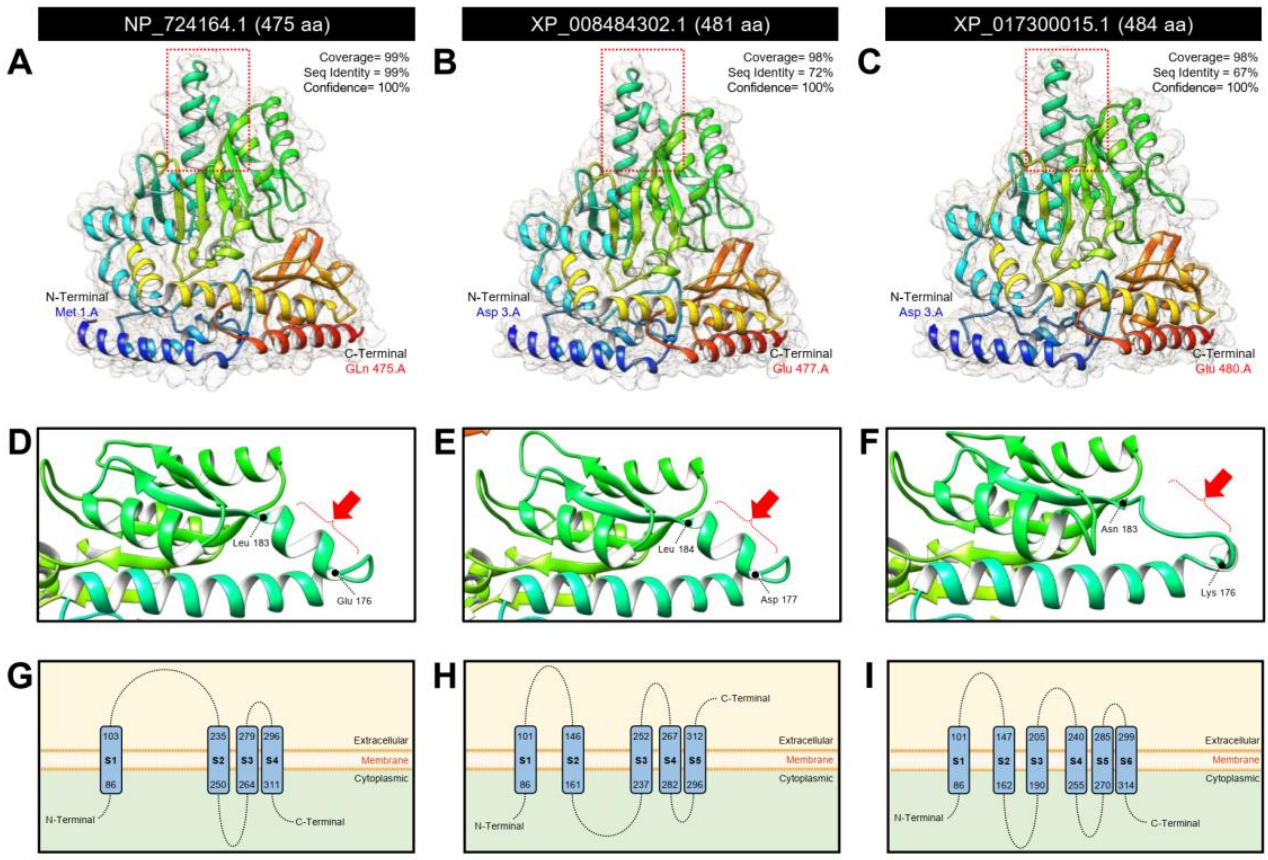

Figure 6. The predicted topology of aromatic L-amino acid decarboxylase (DcAADC) of Diaphorina citri. (A-C) The predicted three-dimensional (3D) monomer structures and their associated mesh surface 
of DmAADC (NP_724164.1), DcAADC-1(XP_008484302.1), and DcAADC-2 (XP_017300015.1), respectively. (D-F) Close-up of the area that outlined with red-dotted lines in panels A, B, and C, respectively. The selected poses were oriented to show one $\alpha$-helix ribbon that was present in $D m A A D C, D c A A D C-1$, but it was absent in DcAADC-2 (pointed by red arrows). Some surrounding AA are shown with their residue number. (G-I) Schematic representation of Phyre2-based predicted topology of $D m A A D C, D c A A D C-1$, and DcAADC-2, respectively. The extracellular and cytoplasmic sides of the membrane are labeled, and the beginning and end of each transmembrane helix illustrated with a number indicating the residue index. Numbers inside the transmembrane (TM) domains (blue rectangle) denote amino acid residues. The predicted topology of TMs have been predicted using the protein homology/analogy recognition engine (Phyre2 web portal-version 2.0) (http:/ / www.sbg.bio.ic.ac.uk/ phyre2/html/page.cgi?id=index, 12 February 2021).

Additionally, the mRNA hairpins of DmAADC (NM_165280.2; 1929 bp), DcAADC-1 (XM_008486080.3; 1939 bp), and DcAADC-2 (XM_017444526.2; 1737 bp) were predicted using the nucleotide sequence with strengths of base pairing probabilities of minimum free energy (MFE; Figure 7A-C, respectively) and centroid secondary structures (Figure 7D-F, respectively). The RNAfold analysis demonstrated that the mRNA hairpins of $D m A A D C$, $D c A A D C-1$, and $D c A A D C-2$ could produce stable MFE secondary structures (MFE $=-682.30$, -569.30 , and $-525.40 \mathrm{Kcal} / \mathrm{mol}$, respectively) and also stable centroid secondary structures $(\mathrm{MFE}=-557.74,-460.40$, and $-426.87 \mathrm{Kcal} / \mathrm{mol}$, respectively). Due to the various sequences size, normalized MFE was calculated. The mRNA hairpin of DcAADC-1 was more stable than other sequences at its MFE (normalized MFE $=-0.2890$; Figure 7B) and centroid (normalized MFE $=-0.2374$; Figure 7E) secondary structures. Moreover, the mountain plot representation of the MFE structure, the centroid structure, the thermodynamic ensemble of RNA structures, and the positional entropy of DmAADC (Figure 7G), DcAADC-1 (Figure $7 \mathrm{H}$ ), and $D c A A D C-2$ (Figure 7I) suggested that all predicted secondary RNA structures were thermodynamically stable. Further, no significant disparities were observed among all predicted mRNA hairpins which can be considered as proof of the stability of the secondary structures.

3.1.3. D. citri Genome Possesses Two Putative Arylalkylamine N-acetyltransferase Genes (DcAANAT)

BLASTp analysis showed that D. citri genome encodes three AA sequences (based on NCBI database, Table S6) and only two protein sequences (based on Diaphorina citri OGS v2.0 proteins dataset, Table S7) with significant similarity to arylalkylamine Nacetyltransferase 1, isoform A (DmAANAT1, also known as DmSNAT; GenBank Accession no. NP_523839.2, 240 aa) from the fruit fly (D. melanogaster). The COBALT-based multiple protein sequences alignment showed that the predicted proteins had relatively high homology with DmAANAT1 protein; however, Dopamine N-acetyltransferase-like, isoform X1 (henceforth DcAANAT-1) encoded by the D. citri locus LOC103507708 (GenBank Accession no. XP_026678312.1; 217 aa) and dopamine $\mathrm{N}$-acetyltransferase-like (henceforth DcAANAT2) encoded by the D. citri locus LOC103507696 (GenBank Accession no. XP_017298946.1; 220 aa) were phylogenetically closer to DmAANAT1 (Figure 8A).

The NCBI protein sequences of DcAANAT-1 and DcAANAT-2 were separately aligned with the sequence of the top-matched proteins from the D. citri database (DcitrP025630.1.1; 217 aa and DcitrP085745.1.1; 240 aa, respectively). The AA alignment showed high similarity and conserved sequence in DcAANAT-1 (Figure S7) but not DcAANAT-2 (Figure S8). Furthermore, the nucleotide sequence of DcAANAT-1 (GenBank Accession no. XM_026822511.1; $1221 \mathrm{bp}$ ) had high similarity and conserved sequences when aligned with the mRNA sequence of DcitrC025630.1.1 (654 bp) from the D. citri database (Figure S9). Low similarity and conserved sequences were observed in DcAANAT-2 (GenBank Accession no. XM_017443457.2; $1911 \mathrm{bp}$ ) when aligned with the mRNA sequence of DcitrP085745.1.1; 240 aa from the D. citri database (Figure S10). 

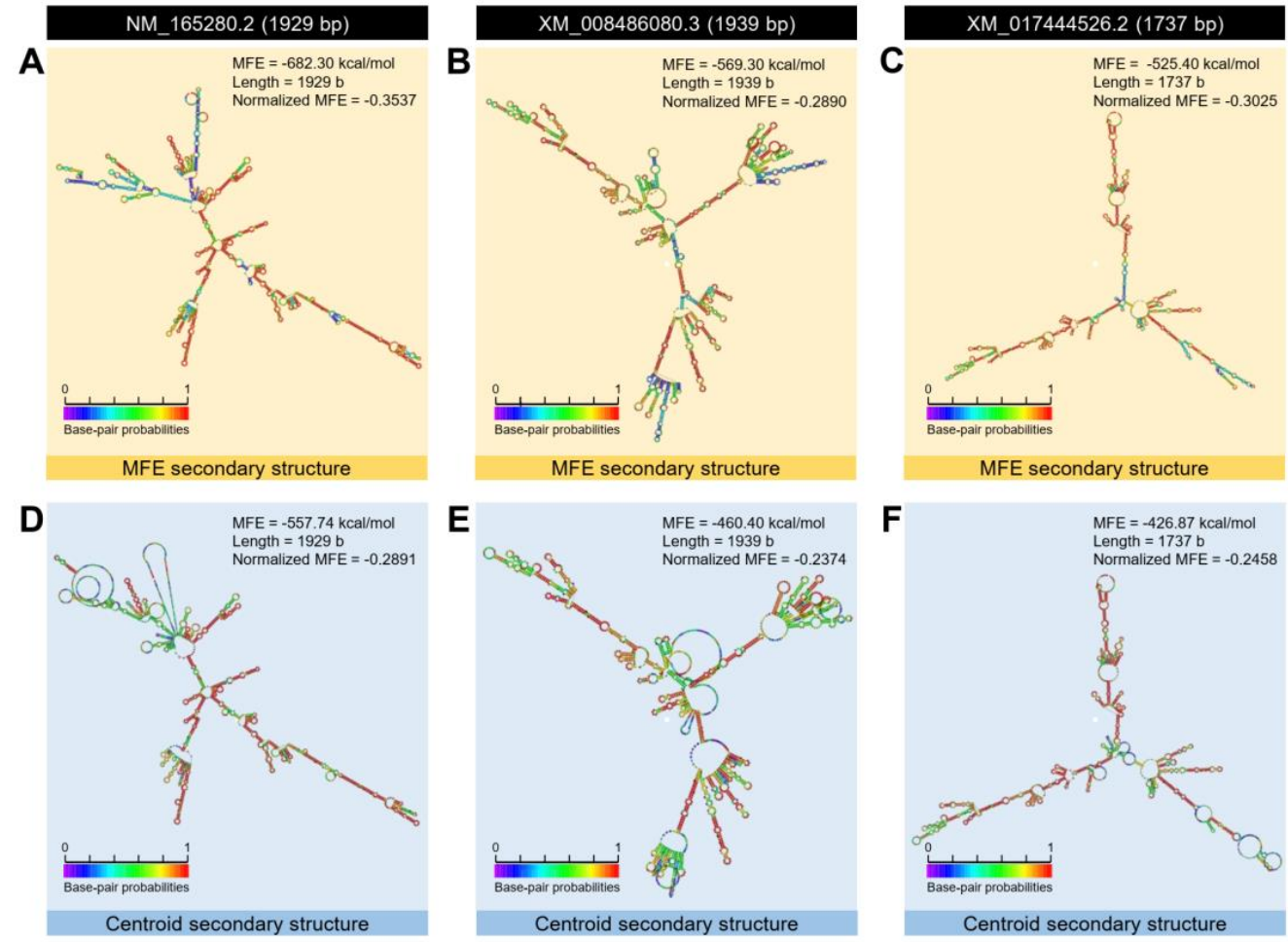

Centroid secondary structure
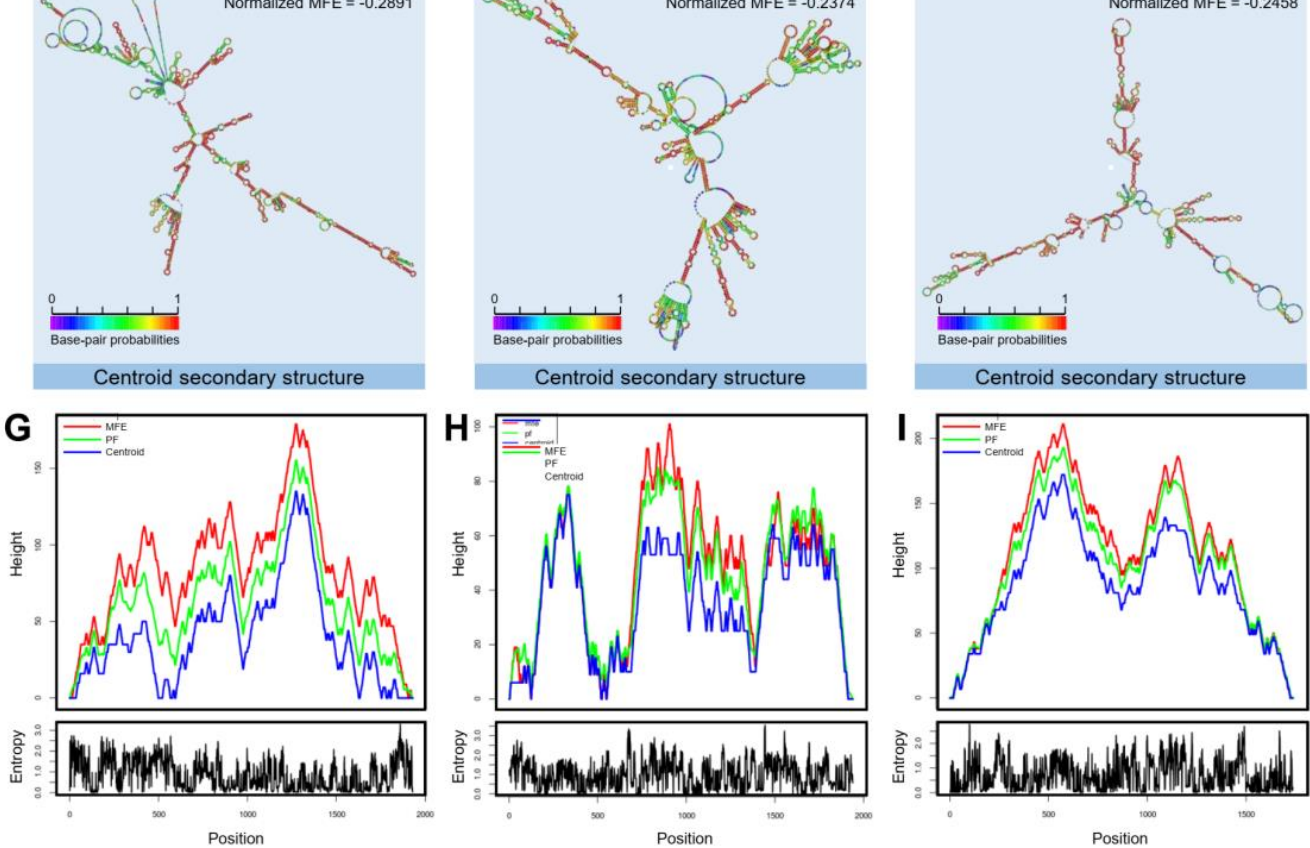

Figure 7. mRNA hairpins of aromatic L-amino acid decarboxylase (DcAADC) of Diaphorina citri. (A-C) Predicted minimum free energy (MFE) secondary structure of DmAADC (NM_165280.2), DcAADC-1 (XM_008486080.3), and DcAADC-2 (XM_017444526.2), respectively. (D-F) Predicted centroid secondary structure of $D m A A D C, D c A A D C-1$, and $D c A A D C-2$, respectively. Colors represent strengths with base-pairing probabilities. (G-I) The mountain plot representations of the MFE structure, the centroid structure, the thermodynamic ensemble of mRNA structures, and the positional entropy of $D m A A D C, D c A A D C-1$, and $D c A A D C-2$, respectively. mRNA secondary structures were predicted using RNAfold web server (http:/ / rna.tbi.univie.ac.at/cgi-bin/RNAWebSuite/RNAfold.cgi, 12 February 2021) using the nucleotide sequences. 
A
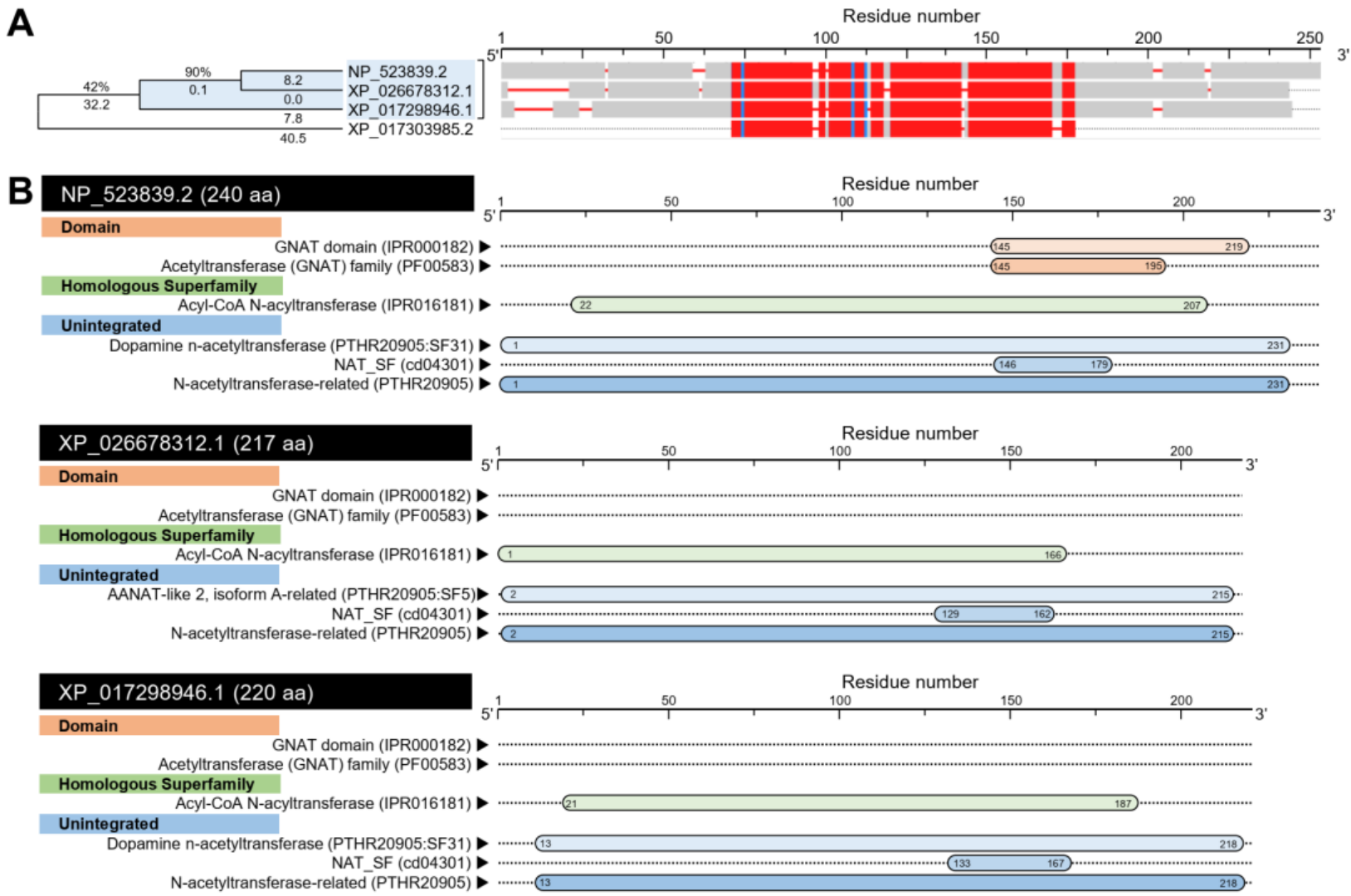

Figure 8. In silico analysis of arylalkylamine N-acetyltransferase (DcAANAT) of Diaphorina citri. (A) Evolutionary analysis using maximum likelihood method and its associated multiple protein sequences alignments using ConstraintBased Alignment tool (COBALT) analysis. The AA sequences were identified using the Protein-Protein BLAST (BLASTp) using arylalkylamine N-acetyltransferase 1, isoform A (DmAANAT; GenBank Accession no. NP_523839.2) from fruit fly, Drosophila melanogaster, as a query sequence, against the Diaphorina citri genome available in GenBank, the national center for biotechnology information website (NCBI, http:/ / www.ncbi.nlm.nih.gov/gene/, 12 February 2021). The tree with the highest log likelihood $(-2252.02)$ is shown. The tree is drawn to scale, with branch lengths measured in the number of substitutions per site (next to the branches). The proportion of sites where at least one unambiguous base is present in at least 1 sequence for each descendent clade is shown next to each internal node in the tree. Evolutionary analyses and the joint tree were conducted in MEGA-X software. In the COBALT analysis, residues were colored using a column-based method according to their relative entropy threshold. Aligned columns with no gaps are colored blue and red, where the red color indicates highly conserved columns and blue indicates less conserved ones. (B) The protein functional and conserved domains analysis of DmAANAT (NP_612080.1), DcAANAT-1 (XP_026678312.1), and DcAANAT-2 (XP_017298946.1) using InterPro Scan tool (https:/ / www.ebi.ac.uk/interpro/, 12 February 2021). GNAT: GCN5-related N-acetyltransferases and NAT-SF: N-acyltransferase superfamily.

InterPro-based analysis of conserved domains suggests a comparable topological similarity between DmAANAT1, DcAANAT-1, and DcAANAT-2 (Figure 8B). All sequences had an acyl-CoA N-acyltransferase (IPR016181) homologous superfamily, and three unintegrated domains including dopamine N-acetyltransferase (PTHR20905:SF31), NAT_SF (cd04301), and N-acetyltransferase-related (PTHR20905) (Figure 8B). However, a Gcn5related N-acetyltransferases (GNAT) domain (IPR000182) was predicted only in DmAANAT1 from $D$. melanogaster, but not in any DcAANAT genes from D. citri (Figure 8B).

The protein tertiary structures of DmAANAT1, DcAANAT-1, and DcAANAT-2 were predicted using the crystal structure of $D$. melanogaster dopamine $\mathrm{N}$-acetyltransferase in complex with CoA and tryptophol (PDB ID: 6k80.1.A) and refined to $1.28 \AA$ resolution with accepted statistics (Figure 9). 

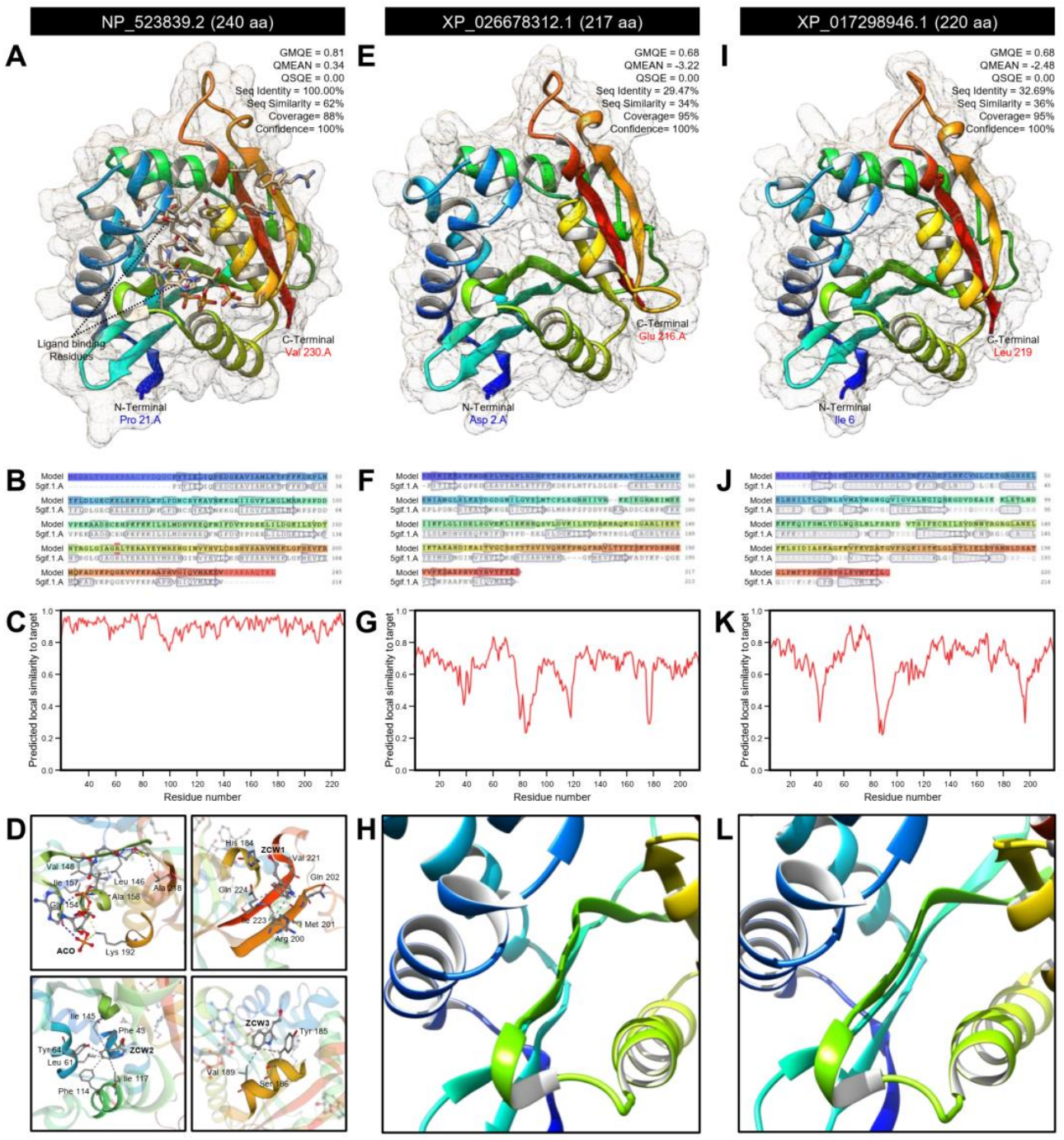

Figure 9. The crystallographic three-dimensional (3D) modeling of arylalkylamine $\mathrm{N}$-acetyltransferase (DcAANAT) of Diaphorina citri. (A,E,I) The predicted three-dimensional (3D) structure model and its associated mesh surface of DmAANAT (NP_612080.1), DcAANAT-1 (XP_026678312.1), and DcAANAT-2 (XP_017298946.1), respectively. The tertiary structures were predicted with $100.0 \%$ confidence by the single highest scoring template of the crystal structure of $D$. melanogaster dopamine $\mathrm{N}$-acetyltransferase in complex with CoA and tryptophol (PDB ID: 6k80.1.A) and refined to $1.28 \AA$ resolution. Protein chains are colored according to the rainbow color spectrum, from blue ( $\mathrm{N}$-terminus) to red (C-terminus). (B,F,J) Model-template alignment of DmAANAT, DcAANAT-1, and DcAANAT-2, respectively. AA sequences of each model were aligned with the template (6k80.1.A). Secondary structures are represented by rectangles ( $\alpha$-helices) and arrows ( $\beta$-sheets). Matched sequences are indicated in black. $(\mathbf{C}, \mathbf{G}, \mathbf{K})$ Local quality estimate of the predicted models of DmAANAT, DcAANAT-1, and DcAANAT-2, respectively. $(\mathbf{D}, \mathbf{H}, \mathbf{L})$ Close-up of the ligand-binding site of DmAANAT, DcAANAT-1, and DcAANAT-2, respectively. The selected poses were oriented to show the entry point for acetyl coenzyme A (ACO) and 2-(1H-indol3-yl)ethanol (ZCW). Some surrounding AA near to the ligand are shown with their residue number. Gray dotted-lines indicate hydrophobic interactions, blue dotted-lines indicate hydrogen bonds, yellow dottedlines indicate salt bridges, and green dotted-lines indicate pi-Stacking. All bioinformatics analyses were carried out based on recent available data on the "Diaphorina citri OGS v2.0 proteins" dataset available on Citrus Greening Solutions website (https: / / citrusgreening.org/organism/Diaphorina_citri/genome, 12 February 2021) and the most recent available data in GenBank, the national center for biotechnology 
information website (NCBI, http:/ / www.ncbi.nlm.nih.gov/gene/, 12 February 2021). The 3D structure was created using the SWISS-MODEL server (https://swissmodel.expasy.org/, 12 February 2021) and visualized using the UCSF-Chimera package (version 1.15) (https:/ / www.cgl.ucsf.edu/ chimera/, 12 February 2021). GMQE: Global model quality estimation and QSQE: Quaternary structure quality estimate.

DmAANAT1 has been predicated as a monomer that covered approximately $88 \%$ of the template protein $(\mathrm{GMQE}=0.81, \mathrm{QMEAN}=0.34, \mathrm{QSQE}=0.00$, seq identity $=100.00 \%$, seq similarity $=62 \%$, and confidence $=100 \%$ ) (Figure 9A). The predicted model was composed of six $\alpha$-helices and ten $\beta$-sheets (Figure 9A,B) with a high local quality estimate (Figure 9C). Further, it had four ligand binding sites for one acetyl coenzyme A (ACO) and three 2-(1H-indol-3-yl)ethanol (ZCW) (Figure 9D). Briefly, 24 residues (Phe 43, Asp 46, Glu 47, Pro 48, Gly 143, Lys 144, Ile 145, Leu 146, Ser 147, Val 148, Arg 153, Gly 154, Leu 155, Gly 156, Ile 157, Ala 158, Val 179, Leu 180, Ser 182, Ser 186, Val 189, and Lys 192) within $4 \AA$ interacted with ACO to form 13 interactions including three hydrophobic interactions (at residues Leu 146, Val 148, and Val 179), eight hydrogen bonds (at residues Leu 146, Leu 146, Val 148, Gly154, Gly154, Gly156, Ile 157, and Ala 158), and two salt bridges with residue Lys 192 (Figure 9D).

Moreover, DmAANAT1 had three ZCW binding sites. ZCW 1 interacts with eight residues within $4 \AA$ including His 184, Arg 200, Met 201, Aln 202, Val 221, Gly 222, Ile 223, and Aln 224 to form four hydrophobic interactions at residues Arg 200, Aln 202, Val 221, and Aln 224 (Figure 9D). ZCW 2 interacts with 12 residues (Phe 43, Glu 47, Asn 50, Leu 61, Tyr 64, Phe 114, Ile 117, Met 121, Lys 144, Ile 145, Leu 180, and Ser 182) within $4 \AA ̊$ to form five hydrophobic interactions (at residues Leu 61, Tyr 64, Phe 114, Ile 117, and Ile 145) and two pi-stacking with Phe 43 (Figure 9D). ZCW 2 interacts with only three residues (Tyr 185,

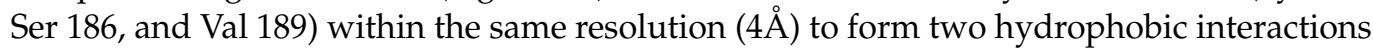
at Tyr 185 and Val 189 residues (Figure 9D).

Correspondingly, roughly 95\% of the DcAANAT-1 sequence (residues Asp 2 to Glu 216) have been modeled with the target protein $(\mathrm{GMQE}=0.68, \mathrm{QMEAN}=-3.22, \mathrm{QSQE}=0.00$, seq identity $=29.47 \%$, seq similarity $=34 \%$, and confidence $=100 \%$ ) (Figure $9 \mathrm{E})$. The predicted model of DcAANAT- 1 contains eight $\alpha$-helix ribbons and eight stranded $\beta$-wings (Figure 9E,F) with a high local quality estimate compared with the target template (Figure 9G). However, no ligand binding sites were observed (Figure $9 \mathrm{H}$ ). Likewise, about $95 \%$ of DcAANAT-2 sequence (residues Ile 6 to Leu 219) were modeled with the target protein (GMQE $=0.68$, QMEAN $=-2.48$, QSQE $=0.00$, seq identity $=32.69 \%$, seq similarity $=36 \%$, and confidence $=100 \%$ ) (Figure 9I). The predicted model of DcAANAT-2 contained seven $\alpha$-helices and eight stranded $\beta$-sheets (Figure 9I,J) with substantial predicted local similarity to target (Figure 9K). Like DcAANAT-1, no ligand binding sites were observed in the model of DcAANAT-2 (Figure 9L).

Furthermore, the mRNA hairpins of DmAANAT1 (NM_079115.3; 1275 bp), DcAANAT-1 (XM_026822511.1; $1221 \mathrm{bp}$ ) and DcAANAT-2 (XM_017443457.2; $1911 \mathrm{bp}$ ) were predicted with strengths of base pairing probabilities of MFE (Figure 10A-C, respectively) and centroid (Figure 10D-F, respectively) secondary structures. RNAfold analysis proved that although the mRNA hairpins of DmAANAT1, DcAANAT-1 and DcAANAT-2 could generate stable MFE secondary structures (MFE $=-393.2,-323.6$, and $-438.6 \mathrm{Kcal} / \mathrm{mol}$, respectively) and centroid secondary structures (MFE $=-119.75,-254.2$, and $-276.97 \mathrm{Kcal} / \mathrm{mol}$, respectively), the MFE and centroid secondary structures of DcAANAT-2 were more stable (normalized MFE $=-0.2295$ and -0.1449 , respectively). Further, the thermodynamic ensemble and the positional entropy of DmAANAT1 (Figure 10G), DcAANAT-1 (Figure 10H), and DcAANAT-2 (Figure 10I) suggested that predicted mRNA hairpins were thermodynamically stable. 

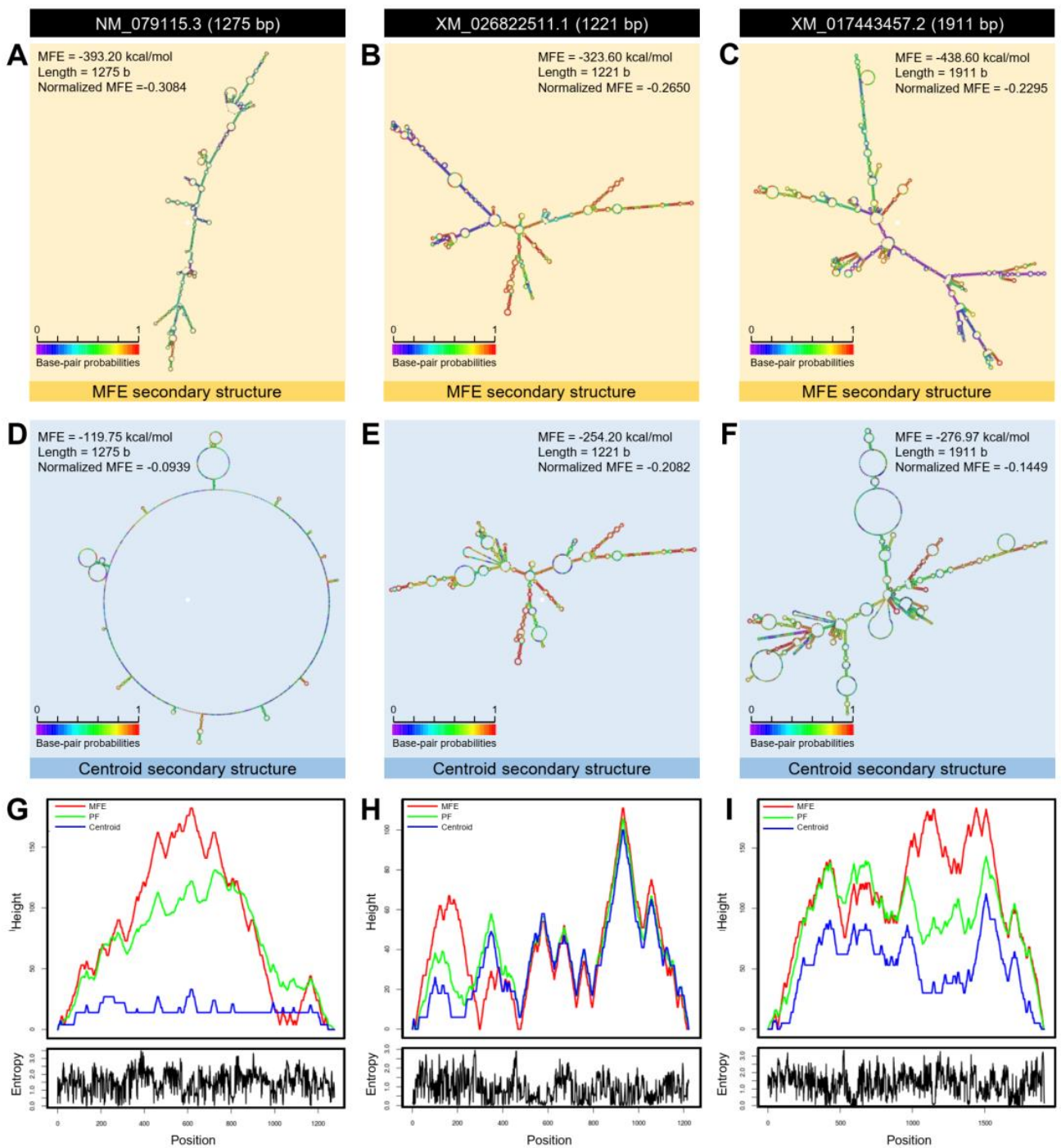

Figure 10. mRNA hairpins of arylalkylamine N-acetyltransferase (DcAANAT) of Diaphorina citri. (A-C) Predicted minimum free energy (MFE) secondary structure of DmAANAT1 (NM_079115.3), DcAANAT-1 (XM_026822511.1), and DcAANAT-2 (XM_017443457.2), respectively. (D-F) Predicted centroid secondary structure of DmAANAT, DcAANAT-1, and DcAANAT-2, respectively. Colors represent strengths with base-pairing probabilities. (G-I) The mountain plot representations of the MFE structure, the centroid structure, the thermodynamic ensemble of mRNA structures, and the positional entropy of DmAANAT, DcAANAT-1, and DcAANAT-2, respectively. mRNA secondary structures were predicted using the RNAfold web server (http:/ /rna.tbi.univie.ac.at/cgibin/RNAWebSuite/RNAfold.cgi, 12 February 2021) using the nucleotide sequences.

3.1.4. D. citri Genome Encodes for a Putative N-acetylserotonin O-methyltransferase (DcASMT)

Digging the D. citri genome using the BLASTp tool retrieved two sequences from the NCBI database (Table S8) and only one sequence from the Diaphorina citri OGS v2.0 proteins dataset (Table S9) that produced significant similarities to $\mathrm{N}$-acetylserotonin O-methyltransferase-like protein, isoform X1 (ClASMT; 260 aa; GenBank Accession no. XP_014251646.1) from bed bug (Cimex lectularius). The multiple protein sequence alignment using COBALT analysis showed that all predicted sequences have relatively high homology with ClASMT protein (Figure 11A). One of these sequences (103 aa; GenBank Accession no. XP_026688590.1) was excluded because it was a partial sequence. However, septum formation protein Maf-like encoded by the D. citri locus LOC113468045 (162 aa; GenBank Accession no. XP_026680472.1) was used for further analysis. Interestingly, this 
AA sequence was highly similar and showed conserved sequences to $\mathrm{N}$-acetylserotonin O-methyltransferase-like protein from the D. citri database (henceforth DcASMT; 274 aa; DcitrP032285.1.1) (Figure S11). Likewise, the nucleotide sequence of DcASMT (746 bp, GenBank Accession no. XM_026824671.1) had high similarity and conserved sequences when aligned with the mRNA sequences from the $D$. citri database (825 bp, DcitrC032285.1.1) (Figure S12). Together, these findings suggest that the DcASMT sequence retrieved from the NCBI database presented the same protein sequence from the $D$. citri database.

A
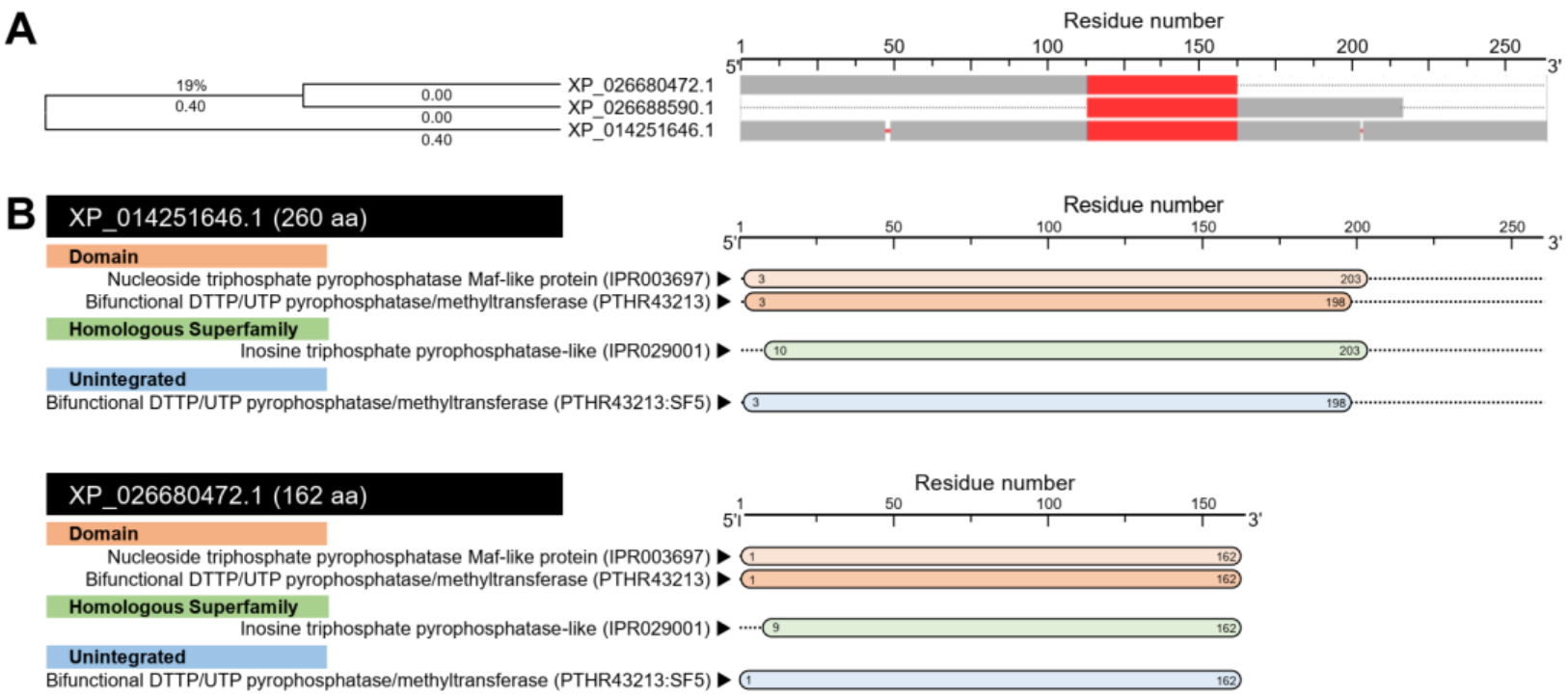

Figure 11. In silico analysis of $N$-acetylserotonin O-methyltransferase (DcASMT) of Diaphorina citri. (A) Evolutionary analysis using maximum likelihood method and its associated multiple protein sequences alignments using ConstraintBased Alignment tool (COBALT) analysis. The AA sequences were identified using the Protein-Protein BLAST (BLASTp) using $\mathrm{N}$-acetylserotonin O-methyltransferase-like protein, isoform X1 (ClASMT; GenBank Accession no. XP_014251646.1) from bed bug (Cimex lectularius) as a query sequence, against Diaphorina citri genome available in GenBank, the national center for biotechnology information website (NCBI, http:/ / www.ncbi.nlm.nih.gov/gene/, 12 February 2021). The tree is drawn to scale, with branch lengths measured in the number of substitutions per site (next to the branches). The proportion of sites where at least one unambiguous base is present in at least 1 sequence for each descendent clade is shown next to each internal node in the tree. Evolutionary analyses and the joint tree were conducted in MEGA-X software. In the COBALT analysis, residues were colored using a column-based method according to their relative entropy threshold. Aligned columns with no gaps are colored blue and red, where the red color indicates highly conserved columns and blue indicates less conserved ones. (B) The protein functional and conserved domains analysis of ClASMT (XP_014251646.1) and DcASMT (XP_026680472.1) using the InterPro Scan tool (https://www.ebi.ac.uk/interpro/, 12 February 2021).

The prediction of the conserved domains using the InterPro Scan tool suggests a high topological similarity among CIASMT and DcASMT (Figure 11B). Both sequences had two domains included nucleoside triphosphate pyrophosphatase Maf-like protein (IPR003697) and bifunctional DTTP/UTP pyrophosphatase/methyltransferase (PTHR43213); a homologous inosine triphosphate pyrophosphatase-like superfamily (IPR029001); and an unintegrated bifunctional DTTP/UTP pyrophosphatase/methyltransferase (PTHR43213:SF5) domain (Figure 11B).

The crystallographic 3D structures of CIASMT and DcASMT were predicted using the crystal structure of the Maf domain of human $\mathrm{N}$-acetylserotonin O-methyltransferase-like protein (PDB ID: 2p5x.2.A) and refined to $2.00 \AA$ A resolution with good statistics (Figure 12). ClASMT was predicted as a monomer that represented approximately $76 \%$ coverage (residues Leu 9 to Asp 203) with remarkable statistics (GMQE =0.58, QMEAN $=-0.06$, QSQE $=0.00$, seq identity $=46.46 \%$, seq similarity $=43 \%$, and confidence $=100 \%$ ) (Figure 12A). The predicted model of ClASMT composed of nine $\alpha$-helices and $11 \beta$-sheets (Figure 12A,B) with good local quality estimate (Figure 12C). Likewise, about 93\% of 
DcASMT sequence (residues Asn 10 to Leu 162) have been modeled with the target protein $(\mathrm{GMQE}=0.74, \mathrm{QMEAN}=0.71, \mathrm{QSQE}=0.00$, seq identity $=44.37 \%$, seq similarity $=41 \%$, and confidence $=100 \%$ ) (Figure 12D). The predicted model of DcASMT contains four $\alpha$-helices and seven stranded $\beta$-sheets (Figure 12D,E) with substantial predicted local similarity to target (Figure 12F).

XP_014251646.1 (260 aa)

A

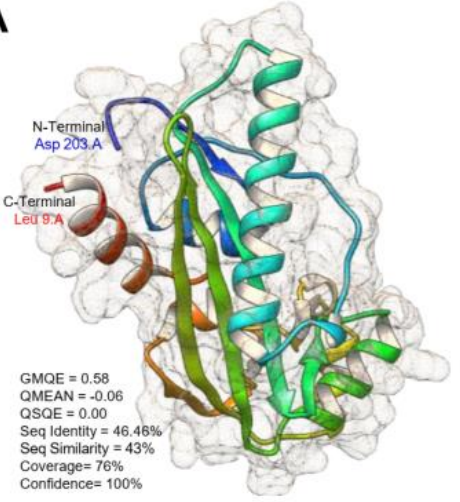

B

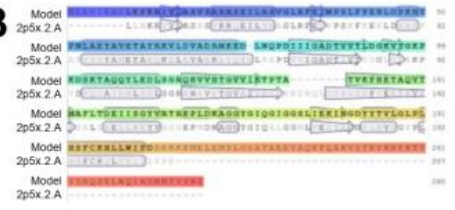

C 兽

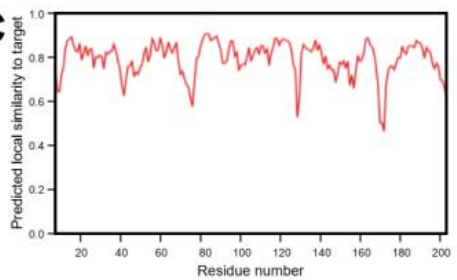

XP 026680472.1 (162 aa)

D
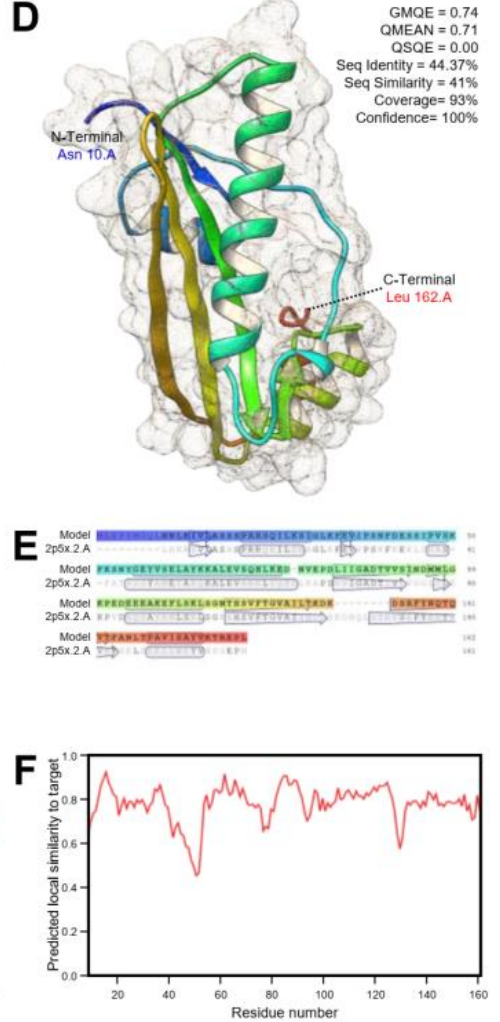

Figure 12. The crystallographic three-dimensional (3D) modeling of $N$-acetylserotonin O-methyltransferase (DcASMT) of Diaphorina citri. (A,D) The predicted three-dimensional (3D) structure model and its associated mesh surface of ClASMT (XP_014251646.1) and DcASMT (XP_026680472.1), respectively. The tertiary structures were predicted with $100.0 \%$ confidence by the single highest scoring template of the crystal structure of the Maf domain of human $\mathrm{N}$-acetylserotonin O-methyltransferase-like protein (PDB ID: 2p5x.2.A) and refined to $2.00 \AA$ resolution. Protein chains are colored according to the rainbow color spectrum, from blue (N-terminus) to red (C-terminus). (B,E) Model-template alignment of ClASMT and DcASMT, respectively. AA sequences of each model were aligned with the template (6k80.1.A). Secondary structures are represented by rectangles ( $\alpha$-helices) and arrows ( $\beta$-sheets). Matched sequences are indicated in black. (C,F) Local quality estimate of the predicted models of ClASMT and DcASMT, respectively. All bioinformatics analyses were carried out based on recent available data on the "Diaphorina citri OGS v2.0 proteins" dataset available on Citrus Greening Solutions website (https:/ / citrusgreening.org/organism/Diaphorina_citri/genome, 12 February 2021) and the most recent available data in GenBank, the national center for biotechnology information website (NCBI, http:/ / www.ncbi.nlm.nih.gov/gene/, 12 February 2021). The 3D structure was created using the SWISS-MODEL server (https:/ / swissmodel.expasy.org/, 12 February 2021) and visualized with the UCSF-Chimera package (version 1.15) (https:/ / www.cgl.ucsf.edu/chimera/, 12 February 2021). GMQE: Global model quality estimation and QSQE: Quaternary structure quality estimate.

Additionally, the mRNA hairpins of ClASMT (1091 bp; XM_014396160.1) and DcASMT (746 bp; GenBank Accession no. XM_026824671.1) were predicted using the nucleotide sequence with strengths of base pairing probabilities of minimum free energy (MFE; Figure 13A,B, 
respectively) and centroid secondary structures (Figure 13C,D, respectively). The RNAfold analysis demonstrated that the mRNA hairpins of DmASMT and DcASMT could produce stable MFE secondary structures (MFE $=-255.50$ and $-190.80 \mathrm{Kcal} / \mathrm{mol}$, respectively) and also stable centroid secondary structures (MFE $=-197.20$ and $-152.04 \mathrm{Kcal} / \mathrm{mol}$, respectively). Moreover, the mountain plot representation of the MFE structure, the centroid structure, the thermodynamic ensemble of RNA structures, and the positional entropy of DmASMT (Figure 13E) and DcASMT (Figure 13F) suggested that both predicted secondary RNA structures were thermodynamically stable.

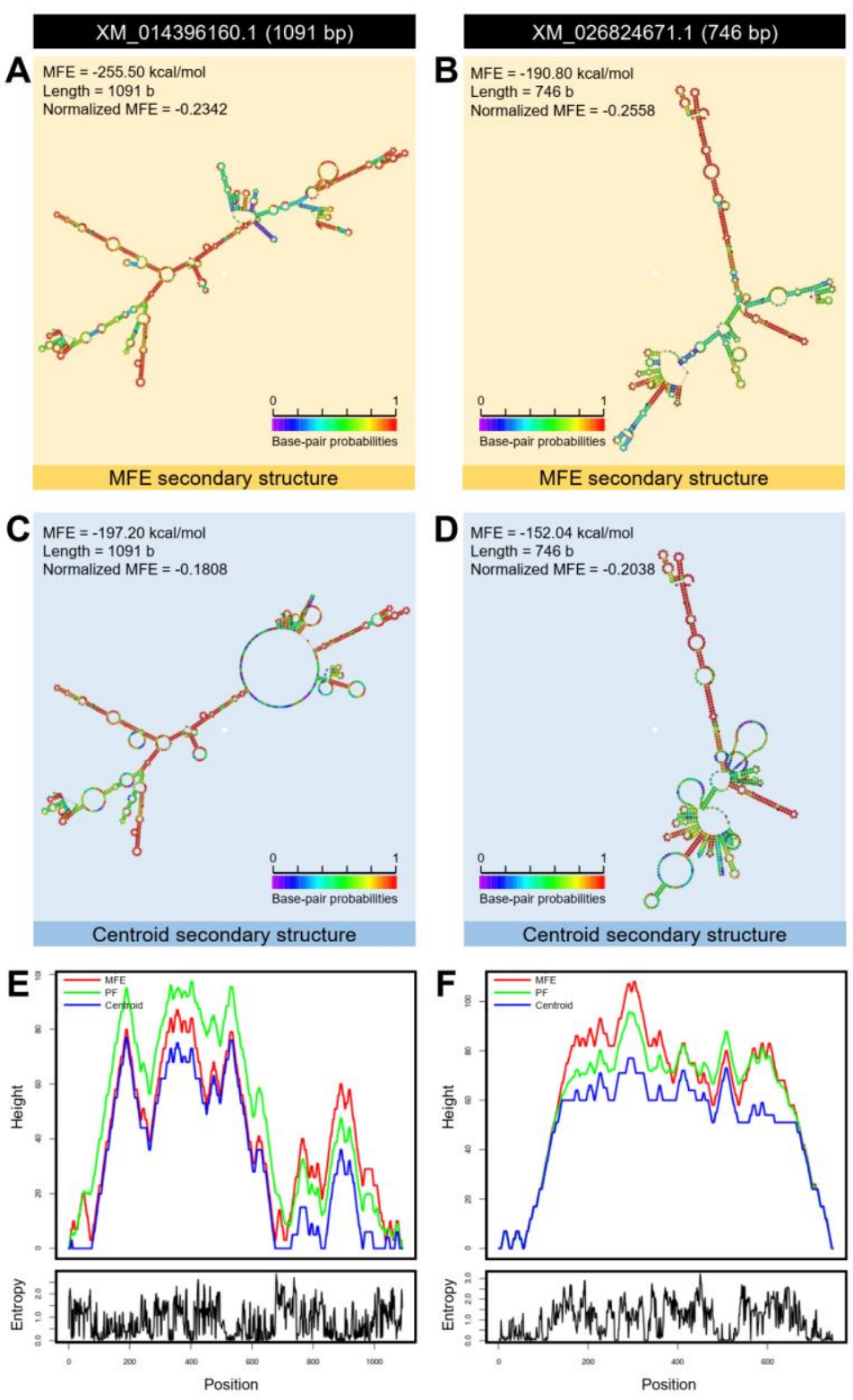

Figure 13. mRNA hairpins of $N$-acetylserotonin O-methyltransferase (DcASMT) of Diaphorina citri. (A,B) Predicted minimum free energy (MFE) secondary structure of ClASMT (XM_014396160.1) and DcASMT (XM_026824671.1), respectively. (C,D) Predicted centroid secondary structure of ClASMT and DcASMT, respectively. Colors represent strengths with base-pairing probabilities. (E,F) The mountain plot representations of the MFE structure, the centroid structure, the thermodynamic ensemble of mRNA structures, and the positional entropy of ClASMT and $D c A S M T$, respectively. mRNA secondary structures were predicted using RNAfold web server (http:/ / rna.tbi.univie.ac.at/cgi-bin/RNAWebSuite/RNAfold.cgi, 12 February 2021) using the nucleotide sequences. 


\subsection{Ca. L. asiaticus Infection Downregulated the Expression of Melatonin Biosynthesis-Related} Genes of D. citri

We investigated the transcript levels of six melatonin biosynthesis-related genes in D. citri (Figure 14). These genes included two DcT5Hs (DcT5H-1 and DcT5H-2), DcAADC, two DcAANATs (DcAANAT-1 and DcAANAT-2), and DcASMT (Figure 14A-F, respectively).
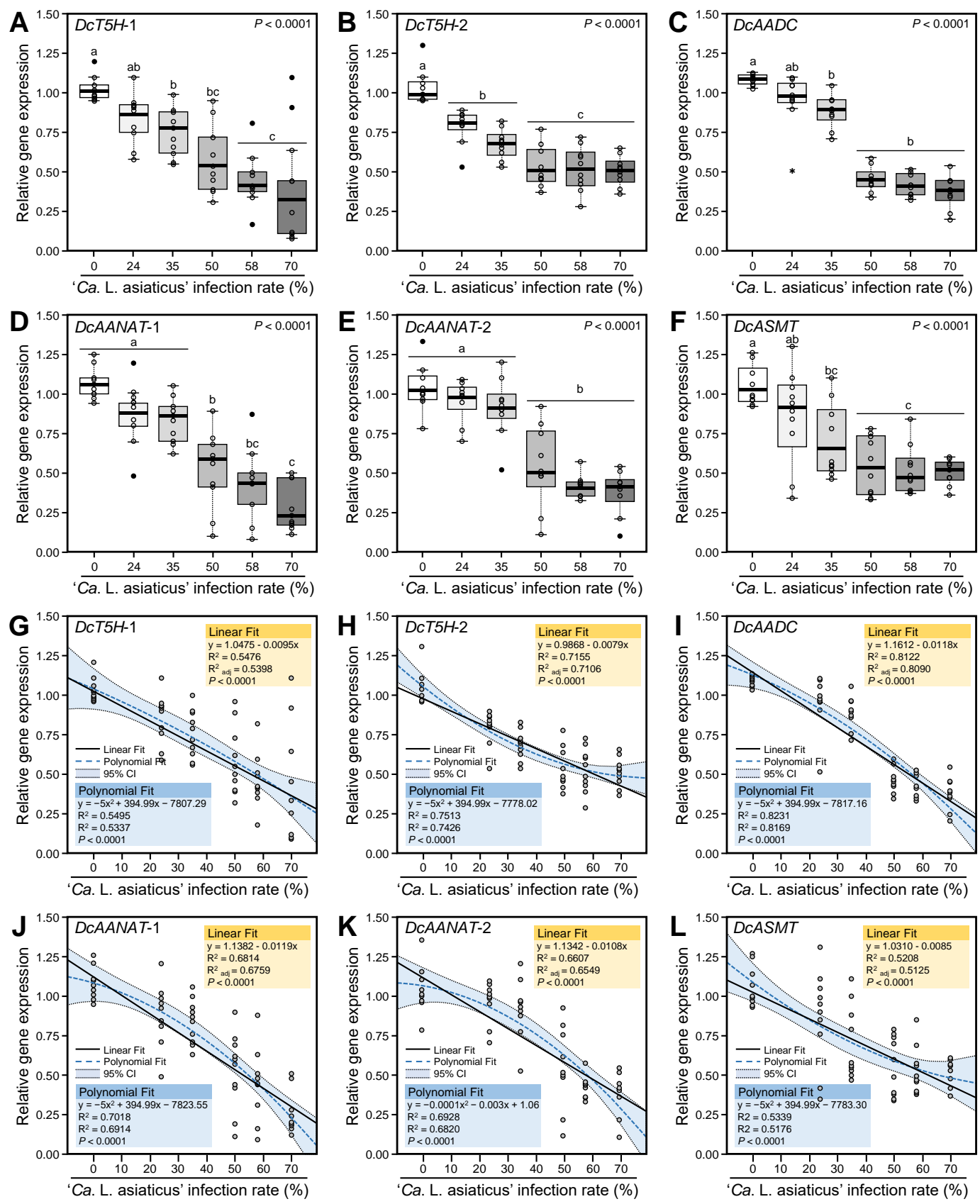

Figure 14. Effect of different infection rates with Candidatus Liberibacter asiaticus on melatonin biosynthesis-related genes of Diaphorina citri. (A-F) Relative gene expression of tryptophan 5-hydroxylase (DcT5H-1 and DcT5H-2), aromatic amino acid decarboxylase $(D c A A D C)$, arylalkylamine $N$-acetyltransferase genes (DcAANAT-1 and DcAANAT-2), and $N$-acetylserotonin O-methyltransferase (DcASMT), respectively. Gene expressions were normalized using two housekeeping genes ( $\alpha$-tubulin and actin), and the changes were analyzed using the $2^{-\Delta \Delta C}$ Tethod. Open dots indicate the raw data $(n=10)$, the black ones indicate potential outliers, whereas horizontal thick lines indicate the medians. Whiskers indicate the minimum, and the maximum values of the data and boxes show the interquartile ranges (twenty-fifth to the seventy-fifth percentile of the data). Different letters indicate statistically significant differences among different infection rates, while the same letter signifies no significant differences between them using Tukey-Kramer honestly significant difference test (Tukey HSD; $p$ <.05). The full list of expressed genes, names, accession numbers, and primers are available in Table 1. (G-L) Simple 
linear regression and quadratic polynomial regression analysis between $\mathrm{C} a$. L. asiaticus infection rates and the expression levels of DcT5H-1, DcT5H-2, DcAADC, DcAANAT-1, DcAANAT-2, and DcASMT, respectively. Open dots present the row data $(n=10)$. The fitted regression line is presented as a black solid-line, while the polynomial regression model is presented as a blue dashed line. The $95 \%$ confidence intervals (95\% CI) for the estimated polynomial regression are blue-shaded and edged by doted-lines. Regression equations, $\mathrm{R}^{2}, \mathrm{R}^{2}$ adj, and $p$-value based on the $\mathrm{F}$ test $(p<0.05)$ were also obtained and presented within the graph.

Our findings showed that the Ca. L. asiaticus infection significantly reduced the transcript levels of all studied melatonin biosynthesis-related genes. The downregulation of melatonin biosynthesis-related genes was proportionally over the $\mathrm{Ca}$. L. asiaticus infection rates. Although the transcript levels of $D c T 5 H-1$ were gradually decreased with $\mathrm{Ca}$. L. asiaticus infection rates, no significant differences were observed between the high Ca. L. asiaticus infection rates (58 and 70\% Ca. L. asiaticus) (Figure 14A). Likewise, Ca. L. asiaticus infection decreased the expression levels of DcT5H-2; however, no significant differences were observed between low infection rates ( 24 and $35 \%$ ), and also no significant differences were observed between the high infection rates (50,58 and $70 \% \mathrm{Ca}$. L. asiaticus) (Figure 14B). Moreover, DcAADC was downregulated in Ca. L. asiaticus-infected D. citri compared with healthy insects, without significant differences among the high infection rates $(50,58$, and $70 \%$ ) (Figure $14 \mathrm{C}$ ). On the other hand, low infection rates (less than $50 \%$ ) did not affect the expression levels of DcAANAT-1 and DcAANAT-2 (Figure 14D,E, respectively). The transcript levels $D c A S M T$ were also gradually reduced under different $\mathrm{Ca}$. L. asiaticus infection rates without significant differences between the high infection rates $(50,58$, and $70 \%)$ (Figure $14 \mathrm{~F})$.

\subsection{Expression Levels of Melatonin Biosynthesis-Related Genes of D. citri Are Negatively Correlated with the Ca. L. asiaticus Infection Rates}

The relationship between the expression levels of melatonin biosynthesis-related genes in $D$. citri and $C a$. L. asiaticus infection rates is presented in Figure 14G-L. The simple linear regression showed that the expression levels of all melatonin biosynthesis-related genes were negatively correlated with the $\mathrm{Ca}$. L. asiaticus infection rates (\%) with moderate to high coefficient of determination $\left(\mathrm{R}^{2}\right)$. The linear regression equations were expressed as follow: DcT5H-1 ( $\mathrm{y}=1.0475-0.0095 \mathrm{x}, \mathrm{R}^{2}=0.5476, \mathrm{R}^{2}{ }_{\text {adj }}=0.5398$, and $p<0.0001$; Figure $\left.14 \mathrm{G}\right)$, DcT5H-2 (y = 0.9868-0.0079x, $R^{2}=0.7155, R^{2}$ adj $=0.7106$, and $p<0.0001$; Figure $\left.14 \mathrm{H}\right)$, $D c A A D C\left(\mathrm{y}=1.1612-0.0118 \mathrm{x}, \mathrm{R}^{2}=0.8122, \mathrm{R}_{\text {adj }}^{2}=0.8090\right.$, and $p<0.0001$; Figure 14I), DcAANAT-1 ( $\mathrm{y}=1.1382-0.0119 \mathrm{x}, \mathrm{R}^{2}=0.6814, \mathrm{R}^{2}$ adj $=0.6759$, and $p<0.0001$; Figure 14J), DcAANAT-2 ( $\mathrm{y}=1.1342-0.0108 \mathrm{x}, \mathrm{R}^{2}=0.6607, \mathrm{R}^{2}$ adj $=0.6549$, and $p<0.0001$; Figure $\left.14 \mathrm{~K}\right)$, and DcASMT ( $\mathrm{y}=1.0310-0.0085 \mathrm{x}, \mathrm{R}^{2}=0.5208, \mathrm{R}^{2}$ adj $=0.5125$, and $p<0.0001$; Figure $14 \mathrm{~L}$ ).

Due to the nonlinear phenomena of the obtained data, the expression levels of melatonin biosynthesis-related genes in $\mathrm{D}$. citri and $\mathrm{Ca}$. L. asiaticus infection rates were fitted with a second-degree polynomial regression model. The polynomial regression models and its associated 95\% confident curves for DcT5H-1 (Figure 14G), DcT5H-2 (Figure 14H), DcAADC (Figure 14I), DcAANAT-1 (Figure 14J), DcAANAT-2 (Figure 14K), and DcASMT (Figure 14L) suggested that the relationship between the expression levels of melatonin biosynthesis-related genes in $\mathrm{D}$. citri and $\mathrm{Ca}$. L. asiaticus infection rates followed a negative and quadratic model with moderate to high $R^{2}$ and $R^{2}$ adj.

\subsection{Melatonin Supplementation Induced the Expression Levels of Melatonin Biosynthesis-Related Genes of D. citri}

We investigated the transcript levels of the melatonin biosynthesis-related genes in healthy and $\mathrm{Ca}$. L. asiaticus-infected $\mathrm{D}$. citri adults after treatment with exogenous melatonin (Figure 15). Generally, the pretreatment with $500 \mu \mathrm{g} \mathrm{mL}{ }^{-1}$ melatonin upregulated the gene expression of all studied genes in both healthy and infected insects. Although the expression levels of melatonin biosynthesis-related genes were lower in Ca. L. asiaticus- 
infected psyllids compared with the control (healthy adults), exogenous melatonin supplementation enhanced the transcript levels of all studied genes to reach the control without significant differences between them (treated-control versus treated-infected psyllids). Accordingly, these findings suggest that melatonin supplementation enhanced the gene expression of melatonin biosynthetic genes of $D$. citri.

A
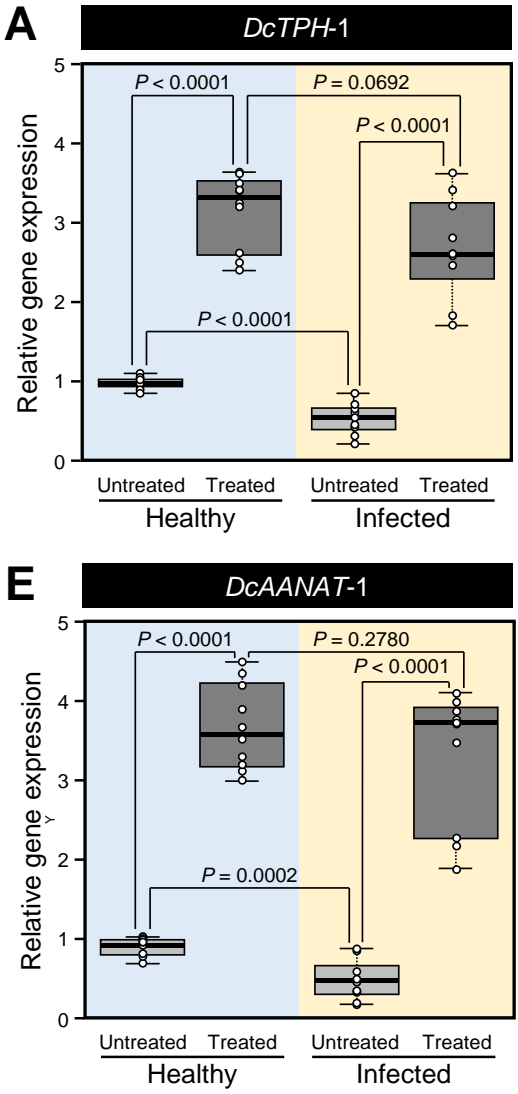

B

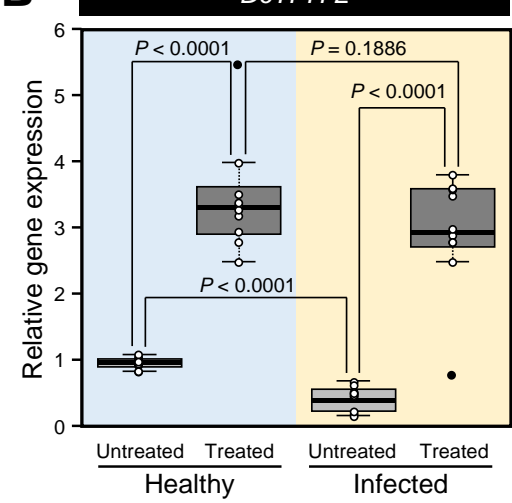

D

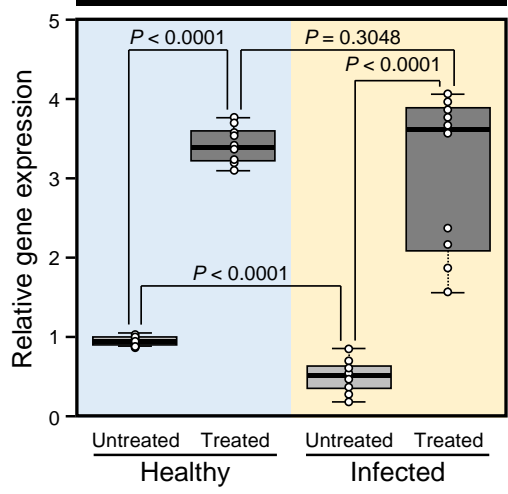

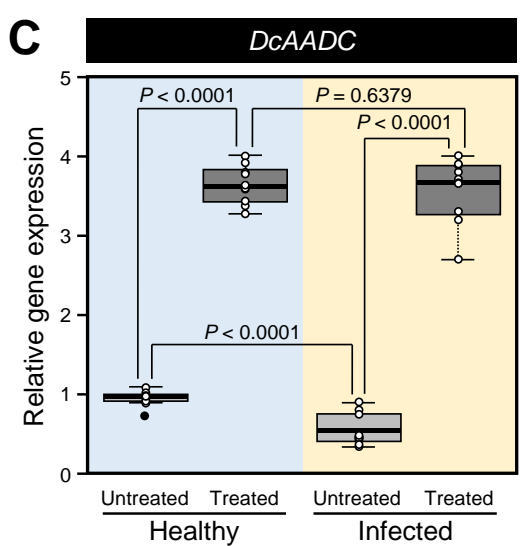

$\mathbf{F}$

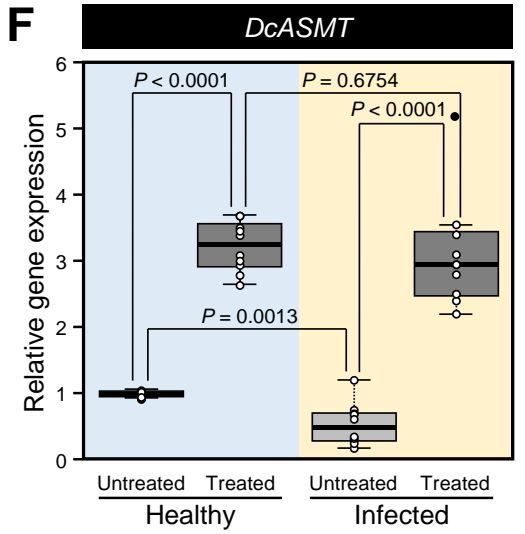

Figure 15. Effect of supplementation on the expression levels of melatonin biosynthesis-related genes of healthy and Ca. L. asiaticus-infected psyllids, Diaphorina citri. (A,B) Relative gene expression of tryptophan 5-hydroxylase (DcT5H-1 and $\mathrm{DcT} 5 \mathrm{H}-2$, respectively), (C) relative gene expression of aromatic amino acid decarboxylase $(D c A A D C),(\mathbf{D}, \mathbf{E})$ relative gene expression of arylalkylamine $\mathrm{N}$-acetyltransferase genes (DcAANAT-1 and DcAANAT-2, respectively), and (F) relative gene expression of $N$-acetylserotonin O-methyltransferase (DcASMT). Gene expressions were normalized using two housekeeping genes ( $\alpha$-tubulin and actin), and the changes were analyzed using the $2^{-\Delta \Delta C} \mathrm{~T}$ method. Open dots indicate the raw data $(n=10)$, the black ones indicate potential outliers, whereas horizontal thick lines indicate the medians. Whiskers indicate the minimum, and the maximum values of the data and boxes show the interquartile ranges (twenty-fifth to the seventy-fifth percentile of the data). Treatments (healthy versus infected and untreated versus treated) were compared using a two-tailed $t$-test, and statistical significance was established as $p<0.05$. The full list of expressed genes, names, accession numbers, and primers are available in Table 1.

\section{Discussion}

Previously, we reported that $\mathrm{Ca}$. L. asiaticus infection significantly reduced the endogenous melatonin content of D. citri [15]. However, it is not clear whether this may happen due to the utilization of insect melatonin directly by $\mathrm{Ca}$. L. asiaticus, or if it was a common cause due to the $C a$. L. asiaticus infection, which might affect the physiological and transcriptional capacities of $D$. citri. The genome sequencing of $C a$. L. asiaticus revealed that it cannot synthesize the amino acid L-tryptophan, the precursor of melatonin [72], from metabolic intermediates [73], and it should acquire it from its host. Further, there is no evidence for melatonin biosynthesis by $\mathrm{Ca}$. L. asiaticus which supports the idea that $\mathrm{Ca}$. L. asiaticus depends on its host (psyllid vectors or citrus plant) for its melatonin needs. 
Nevertheless, recently we showed that melatonin might play an antibacterial role against $\mathrm{Ca}$. L. asiaticus [18], which suggests that utilization of melatonin by Ca. L. asiaticus is not the main reason for melatonin reduction within infected psyllid.

Furthermore, another potential reason for the reduction of melatonin is that it could be a common cause due to the $C a$. L. asiaticus infection, which disrupts the physiological and transcriptional capacities of D. citri [13-15], particularly melatonin biosynthesis-related genes. However, to the best of our knowledge, melatonin biosynthetic genes are not wellannotated yet from the Asian citrus psyllid, D. citri. Previously, we roughly identified four genes to be associated with the melatonin biosynthesis in $D$. citri, which were downregulated in Ca. L. asiaticus-infected psyllids [15]. However, some of these genes have been updated based on the most recent available data in GenBank, the national center for biotechnology information website (NCBI, http:/ /www.ncbi.nlm.nih.gov/gene/, 12 February 2021) including PREDICTED: Diaphorina citri protein henna-like (LOC103524631; GenBank Accession no. XM_017449691.2), PREDICTED: Diaphorina citri tyrosine 3-monooxygenase (LOC103505706; GenBank Accession no. XM_017442547.2), and PREDICTED: Diaphorina citri aromatic L-amino acid decarboxylase-like (LOC103510318; GenBank Accession no. XM_017444526.2), and, as a result of standard genome annotation processing (see www.ncbi.nlm.nih.gov/genome/annotation_euk/process/, 12 February 2021 for more information), even some sequences were removed including PREDICTED: Diaphorina citri dopamine N-acetyltransferase-like (LOC103507708; GenBank Accession no. XM_008472208.2) and PREDICTED: Diaphorina citri arf-GAP domain and FG repeatcontaining protein 1 (LOC103510708; GenBank Accession no. XM_017444656.1).

Therefore, herein, we carried out a comprehensive in silico and bioinformatics analysis to deeply identify the melatonin biosynthesis-related genes of $D$. citri using two major databases including the D. citri-specific dataset of "Diaphorina citri OGS v2.0 proteins" available on Citrus Greening Solutions website (https:/ / citrusgreening.org/organism/ Diaphorina_citri/genome, 12 February 2021) [58] and the most popular database of GenBank, the national center for biotechnology information website (NCBI, http:/ / www.ncbi. nlm.nih.gov/gene/, 12 February 2021). A proposed melatonin biosynthesis pathway and its associated genes in D. citri is presented in Figure 16.

Briefly, we suggest that melatonin in $D$. citri is synthesized from the ${ }_{\mathrm{L}}$-tryptophan via four enzymatic steps. The first step is the oxidation of $\mathrm{L}_{\mathrm{L}}$-tryptophan to 5-hydroxytryptophan [74] via the activity of $D c T 5 H$. Our findings showed that the $D$. citri genome possesses at least two sequences that were relatively homologous and were phylogenetically closer to $\mathrm{DmT5H}$ from D. melanogaster [48,49] including putative tryptophan 5-hydroxylase 1-like (DcT5H-1; DcitrP076520.1.1) and protein henna (DcT5H-2; DcitrP012845.1.1). T5H is the rate-limiting enzyme in serotonin biosynthesis $[28,29]$ that works in the presence of molecular oxygen and requires tetrahydrobiopterin as a cofactor.

Our InterPro-based analysis showed that both DcT5Hs have a tetrahydrobiopterindependent aromatic amino acid (ArAA hydroxylase) family [75] that catalyzes ring hydroxylation of aromatic amino acids, using tetrahydrobiopterin (BH4) as a substrate. Previous studies showed that all eukaryotic $\mathrm{T} 5 \mathrm{H}$ are homotetramers and include a regulatory $\mathrm{N}$-terminal domain, a catalytic domain, and a C-terminal oligomerization motif [76,77]. In agreement with these studies, the crystallographic 3D structures of both DcT5Hs modeled with the crystal structure of human tryptophan hydroxylase 2 (TPH2), catalytic domain (4v06.1.A) [78], encompassed two close conserved histidines that are involved in the binding to iron [79] and one more distant acidic residue, usually glutamic acid in our predicted models. This arrangement of ligands suggests that DcT5Hs are metalloproteins. Interestingly, the expression levels of both DcT5Hs were decreased in Ca. L. asiaticus-infected psyllids and showed an almost identical profile with endogenous melatonin content from our previous study [15]. Collectively, these findings suggest that DcT5Hs (DcT5H-1 and DcT5H-2) could play a key role in melatonin biosynthesis. However, further studies are required to clarify the functional and/or regulatory roles of DcT5Hs in D. citri. 


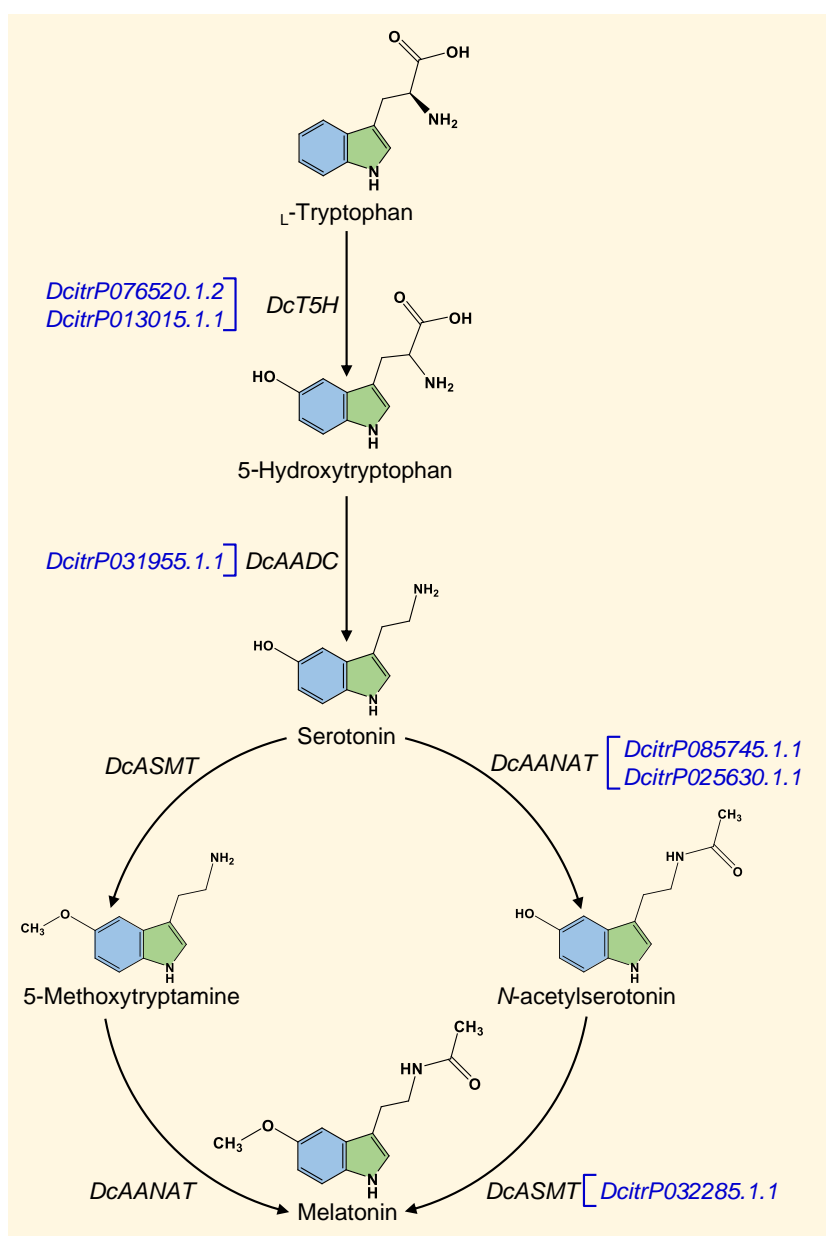

Figure 16. Proposed melatonin biosynthesis pathway in Diaphorina citri and its associated genes. The listed putative gene candidates were identified based on the top-matches of recent available data on the "Diaphorina citri OGS v2.0 proteins" dataset available on the citrus greening solutions website (https:/ / citrusgreening.org/organism/Diaphorina_citri/genome, 12 February 2021). DcT5H: Tryptophan 5-hydroxylase, DcAADC: Aromatic amino acid decarboxylase, DcAANAT: Arylalkylamine $\mathrm{N}$-acetyltransferase, and DcASMT: $\mathrm{N}$-acetylserotonin O-methyltransferase.

The second step in the melatonin biosynthesis pathway is the decarboxylation of 5-hydroxytryptophan to the indoleamine serotonin using the pyridoxal 5-phosphate (PLP)-dependent enzyme aromatic L-amino acid decarboxylase (AADC) [28,40]. Our findings showed that the $D$. citri genome could encode for a putative Dopa decarboxylase (DcDDC; DcitrP031955.1.1) that produced significant similarities to DmDDC (also known as $D m A A D C$ ) from $D$. melanogaster [50-52]. This protein was described in the current study as $D c A A D C$, indicating its aromatic L-amino acid decarboxylase activity. Based on the InterPro analysis, this enzyme is a pyridoxal phosphate-dependent decarboxylase that belongs to the group II decarboxylases $[80,81]$ and shares a region of a conserved lysine residue, which provides the binding site for the PLP group [80,82]. Further, InterPro analysis showed that $D C A A D C$ seems to share regions of sequence similarity with aromatic-L-amino acid decarboxylase (DDC; also known as L-dopa decarboxylase or tryptophan decarboxylase), glutamate decarboxylase $(G A D)$, histidine decarboxylase $(H D C)$, and tyrosine decarboxylase (TyrDC) [80-83] since it had a DDC/GAD/HDC/TyrDC pyridoxal-phosphate attachment site. These enzymes are collectively known as group II decarboxylases [81].

Moreover, our findings suggest that $D c A A D C$ might act as an amino acid-transporter and could be essential for primary carbon metabolism. Although the homo-oligomerization state of AADC proteins has not been extensively investigated, our findings suggest that $D c A A D C$ might form a homodimer in the membrane; nevertheless, previous studies suggest 
that this is dispensable for transport [84]. Our findings showed that $\mathrm{Ca}$. L. asiaticus infection diminished the transcript levels of $D c A A A D$ of $D$. citri compared to uninfected psyllids. The reduction in the $D c A A A D$ expression was consistent with the profile of endogenous melatonin upon different $C a$. L. asiaticus infection rates as reported in our previous study [15]. Moreover, melatonin supplementation enhanced the DcAADC expression and reverses the negative effects of $C a$. L. asiaticus. Taken together, these findings suggest that $D C A A D C$ might play a dual role in melatonin biosynthesis and amino acid transportation. However, further studies are required to investigate the functional and/or regulatory roles of DcAADC in D. citri.

The third step in the melatonin biosynthesis pathway is the $\mathrm{N}$-acetylation of serotonin to form $\mathrm{N}$-acetylserotonin using serotonin $\mathrm{N}$-acetyltransferase (SNAT; also known as arylalkylamine $N$-acetyltransferase [AANAT]) [29]. Our findings showed that the D. citri genome possesses two putative dopamine $N$-acetyltransferase proteins (DcitrP025630.1.1 and DcitrP085745.1.1) with significant similarity to arylalkylamine $N$-acetyltransferase 1 , isoform A (DmAANAT1) from D. melanogaster [53-55]. Both proteins were described in the current study as arylalkylamine $N$-acetyltransferase (DcAANAT-1 and DcAANAT-2) indicating their $\mathrm{N}$-acetylation activity. These findings are in agreement with studies on $D$. melanogaster where two AANAT variants (AANATA and AANATB) were identified $[59,85]$. Although both variants were physiologically relevant in D. melanogaster, they were differentially expressed with respect to tissue distribution and developmental stages $[59,85]$. In contrast, the expression patterns of DcAANAT-1 and DCAANAT-2 in the current study were almost identical with slight differences which suggest that both enzymes serve the same metabolic role in $D$. citri.

AANAT is an acetyl-CoA-dependent enzyme that belongs to GCN5 N-acetyltransferases (GNATs) family [86]. It is the penultimate enzyme in melatonin biosynthesis that catalyzes the transfer of the acetyl group of acetyl-CoA to the primary amine of serotonin to form $\mathrm{N}$-acetylserotonin and CoA [86]. In the current study, InterPro analysis showed that both DcAANAT-1 and DcAANAT-2 have a structural domain of Acyl-CoA N-acyltransferase homologous superfamily. This domain has a triple-layer $\alpha / \beta / \alpha$ structure that contains mixed $\beta$-sheets. The crystallographic 3D structures showed that DcAANAT-1 and DcAANAT-2 have three antiparallel $\beta$-strands like DmAANAT. Although coenzyme A binding pockets were observed via the InterPro analysis, no acyl-CoA binding sites were observed in the tertiary structures of DcAANAT-1, and DcAANAT-2. Our findings in the current study showed that low infection rates of Ca. L. asiaticus (less than 50\%) did not affect the expression levels of DcAANAT-1 and DcAANAT-2; however, our previous study showed that low infection rates significantly reduced the endogenous melatonin content [15]. On the other hand, the higher infection rates reduced the expression levels of both genes in agreement with the endogenous melatonin profile of our previous study [15]. Together, these findings suggest that DcAANAT may not be a rate-determining enzyme in melatonin biosynthesis in D. citri. However, further studies are required to investigate the functional and/or regulatory roles of DcAANAT in D. citri.

Finally, N-acetylserotonin is subsequently methylated by $N$-acetylserotonin O-methyltransferase (ASMT) as a final step in the melatonin production [28,30-32]. Our findings showed that the D.citri genome encodes for a putative $N$-acetylserotonin O-methyltransferase-like (DCASMT; DcitrP032285.1.1) that is producing significant similarities to $\mathrm{N}$-acetylserotonin O-methyltransferase-like protein, isoform X1 (ClASMT) from bed bug (C. lectularius). Herein, we used CIASMT from bed bug as a query sequence because, to the best of our knowledge, no ASMT genes have been identified from $D$. melanogaster yet. Like other melatonin biosynthesis-related genes in this study, DcASMT was significantly downregulated in Ca. L. asiaticus-infected psyllids and agreed with the melatonin profile in our previous study [15].

Moreover, we proposed an alternative route for melatonin biosynthesis from serotonin (Figure 16). Briefly, we suggest that serotonin could be methylated first, rather than $\mathrm{N}$-acetylated as discussed above, to form 5-methoxytryptamine using ASMT. Subsequently, 
5-methoxytryptamine is $\mathrm{N}$-acetylated to form melatonin using SNAT. However, a previous study showed that the enzymatic activity of ASMT was about 14-times greater when it reacted with $\mathrm{N}$-acetylserotonin than when it reacted with serotonin [87] which suggests methylation, but not $\mathrm{N}$-acetylation, of $\mathrm{N}$-acetylserotonin, as the last step of melatonin. Nevertheless, this alternative route might occur under specific circumstances [31,33]. As summarized above, melatonin biosynthesis is controlled by four successive enzymes; however, SNAT was proposed to be the key rate-limiting enzyme in this pathway in vertebrates [28], but not at night [88]. Instead, ASMT may be a rate-limiting enzyme during the nocturnal production of melatonin [88].

\section{Conclusions}

In conclusion, in the present study, we computationally identified six melatonin biosynthesis-related gene candidates (two DcT5Hs, one DcAADC, two DcAANATs, and one DcASMT) in D. citri. These genes were definitionally expressed within the adults of $D$. citri after challenging with gradient infection rates of the phytopathogenic bacterium $\mathrm{Ca}$. L. asiaticus. Moreover, the expression patterns of these genes demonstrated a piece of indirect evidence for the return of melatonin to its normal levels in Ca. L. asiaticusinfected psyllids after melatonin supplementation and confirmed the association of these genes with the melatonin biosynthesis pathway. However, further investigations are required to explore the functional and/or regulatory roles of these genes in melatonin biosynthesis. Our findings of this study are a further step for optimization and cloning of melatonin biosynthesis genes of $D$. citri. They could rapidly be identified via in silico analysis and subsequently subjected to in vitro and in vivo confirmatory studies, since our previous study showed that inhibition of melatonin biosynthesis was associated with reduced longevity of $D$. citri [15]. Therefore, the identified genes in this study could be good candidates that serve as potential targets for RNA interference (RNAi)-based control or other sustainable control strategies of $D$. citri.

Supplementary Materials: The following supplementary materials are available online at https: //www.mdpi.com/article/10.3390/insects12040317/s1, Table S1. Primers used for gene expression analysis of melatonin-biosynthetic enzymes of $D$. citri by real-time RT-PCR. Table S2. Sequences from Diaphorina citri that produce significant alignments with tryptophan hydroxylase from Drosophila melanogaster (T5H; GenBank Accession no. NP_612080.1) using the NCBI database. Table S3. Sequences from Diaphorina citri that produce significant alignments with tryptophan hydroxylase from Drosophila melanogaster (T5H; GenBank Accession no. NP_612080.1) using the "Diaphorina citri OGS v2.0 proteins" BLAST dataset. Table S4. Sequences from Diaphorina citri that produce significant alignments with dopa decarboxylase, isoform B (GenBank Accession no. NP_724164.1) from Drosophila melanogaster using the NCBI database. Table S5. Sequences from Diaphorina citri that produce significant alignments with dopa decarboxylase, isoform B (GenBank Accession no. NP_724164.1) from Drosophila melanogaster using the "Diaphorina citri OGS v2.0 proteins" BLAST dataset. Table S6. Sequences from Diaphorina citri that produce significant alignments with arylalkylamine N-acetyltransferase 1, isoform A (AANAT1; GenBank Accession no. NP_523839.2) from Drosophila melanogaster using the NCBI database. Table S7. Sequences from Diaphorina citri that produce significant alignments with arylalkylamine N-acetyltransferase 1, isoform A (AANAT1; GenBank Accession no. NP_523839.2) from Drosophila melanogaster using the "Diaphorina citri OGS v2.0 proteins" BLAST dataset. Table S8. Sequences from Diaphorina citri that produce significant alignments with $\mathrm{N}$-acetylserotonin O-methyltransferase-like protein (ASMT; GenBank Accession no. XP_014251646.1) from bed bug (Cimex lectularius) using the NCBI database. Table S9. Sequences from Diaphorina citri that produce significant alignments with $\mathrm{N}$-acetylserotonin O-methyltransferase-like protein (ASMT; GenBank Accession no. XP_014251646.1) from bed bug (Cimex lectularius) using the "Diaphorina citri OGS v2.0 proteins" BLAST dataset. Figure S1. Multiple AA sequence alignment of DcT5H-1 from Diaphorina citri. Figure S2. Multiple AA sequence alignment of DcT5H-2 from Diaphorina citri. Figure S3. Multiple nucleotide sequence alignment of DcT5H-1 from Diaphorina citri. Figure S4. Multiple nucleotide sequence alignment of DcT5H-2 from Diaphorina citri. Figure S5. Multiple AA sequence alignment of DCAADC from Diaphorina citri. Figure S6. Multiple nucleotide sequence alignment of DcT5H-1 from Diaphorina citri. Figure S7. Multiple AA sequence alignment of 
DcAANAT-1 from Diaphorina citri. Figure S8. Multiple AA sequence alignment of DcAANAT-2 from Diaphorina citri. Figure S9. Multiple nucleotide sequence alignment of DcAANAT-1 from Diaphorina citri. Figure S10. Multiple nucleotide sequence alignment of DcAANAT-2 from Diaphorina citri. Figure S11. Multiple AA sequence alignment of DcASMT from Diaphorina citri. Figure S12. Multiple nucleotide sequence alignment of DcASMT from Diaphorina citri.

Author Contributions: N.K., together with Y.N., conceptualized the study and contributed to the experimental design. Y.N. and N.K. contributed with analytic tools, data analysis, and finalized the figures. Finally, N.K. and Y.N. wrote and finalized the manuscript. All authors have read and agreed to the published version of the manuscript.

Funding: This work was generously supported by grant No. 201500955-04 for NK from SCRI, NIFA-USDA.

Institutional Review Board Statement: Not applicable.

Data Availability Statement: Data will be shared upon request to the corresponding author.

Acknowledgments: The authors would like to acknowledge the members of our laboratory for helpful discussions, detailed comments, and constructive suggestions. 3D-molecular graphics and analyses performed with UCSF Chimera, developed by the Resource for Biocomputing, Visualization, and Informatics at the University of California, San Francisco, with support from NIH P41-GM103311.

Conflicts of Interest: The authors declare that the research was conducted in the absence of any commercial or financial relationships that could be construed as a potential conflict of interest.

\section{References}

1. Bové, J.M. Huanglongbing: A destructive, newly-emerging, century-old diesease of citrus. J. Plant Pathol. 2006, 88, 7-37.

2. Gottwald, T.R. Current epidemiological understanding of citrus Huanglongbing. Annu. Rev. Phytopathol. 2010, 48, 119-139. [CrossRef] [PubMed]

3. Wang, N.; Trivedi, P. Citrus huanglongbing: A newly relevant disease presents unprecedented challenges. Phytopathology 2013, 103, 652-665. [CrossRef] [PubMed]

4. Nehela, Y.; Killiny, N. Revisiting the complex pathosystem of huanglongbing: Deciphering the role of citrus metabolites in symptom development. Metabolites 2020, 10, 409. [CrossRef]

5. Singerman, A.; Rogers, M.E. The economic challenges of dealing with citrus greening: The case of Florida. J. Integr. Pest Manag. 2020, 11, pmz037. [CrossRef]

6. Grafton-Cardwell, E.E.; Stelinski, L.L.; Stansly, P.A. Biology and management of Asian citrus psyllid, vector of the huanglongbing pathogens. Annu. Rev. Entomol. 2013, 58, 413-432. [CrossRef] [PubMed]

7. Milosavljevic, I.; Schall, K.; Hoddle, C.; Morgan, D.; Hoddle, M. Biocontrol program targets Asian citrus psyllid in California's urban areas. Calif. Agric. 2017, 71, 169-177. [CrossRef]

8. Milne, A.E.; Gottwald, T.; Parnell, S.R.; Alonso Chavez, V.; van den Bosch, F. What makes or breaks a campaign to stop an invading plant pathogen? PLOS Comput. Biol. 2020, 16, e1007570. [CrossRef]

9. Halbert, S.E.; Manjunath, K.L. Asian citrus psyllids (Sternorrhyncha: Psyllidae) and greening disease of citrus: A literature review and assessment of risk in Florida. Florida Entomol. 2004, 87, 330-353. [CrossRef]

10. Pelz-Stelinski, K.S.; Killiny, N. Better together: Association with 'Candidatus liberibacter asiaticus' increases the reproductive fitness of its insect vector, Diaphorina citri (Hemiptera: Liviidae). Ann. Entomol. Soc. Am. 2016, 48, 539-548. [CrossRef] [PubMed]

11. Tiwari, S.; Pelz-Stelinski, K.; Stelinski, L.L. Effect of Candidatus Liberibacter asiaticus infection on susceptibility of Asian citrus psyllid, Diaphorina citri, to selected insecticides. Pest Manag. Sci. 2011, 67, 94-99. [CrossRef]

12. Martini, X.; Hoffmann, M.; Coy, M.R.; Stelinski, L.L.; Pelz-Stelinski, K.S. Infection of an insect vector with a bacterial plant pathogen increases its propensity for dispersal. PLoS ONE 2015, 10, e0129373. [CrossRef] [PubMed]

13. Lu, Z.; Killiny, N. Huanglongbing pathogen Candidatus Liberibacter asiaticus exploits the energy metabolism and host defence responses of its vector Diaphorina citri. Physiol. Entomol. 2017, 42, 319-335. [CrossRef]

14. Killiny, N.; Nehela, Y.; Hijaz, F.; Vincent, C.I. A plant pathogenic bacterium exploits the tricarboxylic acid cycle metabolic pathway of its insect vector. Virulence 2018, 9, 99-109. [CrossRef] [PubMed]

15. Nehela, Y.; Killiny, N. Infection with phytopathogenic bacterium inhibits melatonin biosynthesis, decreases longevity of its vector, and suppresses the free radical-defense. J. Pineal Res. 2018, 65, e12511. [CrossRef] [PubMed]

16. Reiter, R.J. Melatonin: The chemical expression of darkness. Mol. Cell. Endocrinol. 1991, 79, C153-C158. [CrossRef]

17. Ikegami, K.; Yoshimura, T. Circadian clocks and the measurement of daylength in seasonal reproduction. Mol. Cell. Endocrinol. 2012, 349, 76-81. [CrossRef]

18. Nehela, Y.; Killiny, N. Melatonin is involved in citrus response to the pathogen huanglongbing via modulation of phytohormonal biosynthesis. Plant Physiol. 2020, 184, 2216-2239. [CrossRef] [PubMed] 
19. Cipolla-Neto, J.; Amaral, F.G.; Afeche, S.C.; Tan, D.X.; Reiter, R.J. Melatonin, energy metabolism, and obesity: A review. J. Pineal Res. 2014, 56, 371-381. [CrossRef] [PubMed]

20. Galano, A.; Tan, D.X.; Reiter, R.J. Melatonin as a natural ally against oxidative stress: A physicochemical examination. J. Pineal Res. 2011, 51, 1-16. [CrossRef] [PubMed]

21. Carrillo-Vico, A.; Lardone, P.J.; Alvarez-Sánchez, N.; Rodríguez-Rodríguez, A.; Guerrero, J.M. Melatonin: Buffering the immune system. Int. J. Mol. Sci. 2013, 14, 8638-8683. [CrossRef] [PubMed]

22. Tarocco, A.; Caroccia, N.; Morciano, G.; Wieckowski, M.R.; Ancora, G.; Garani, G.; Pinton, P. Melatonin as a master regulator of cell death and inflammation: Molecular mechanisms and clinical implications for newborn care. Cell Death Dis. 2019, 10, 1-12. [CrossRef]

23. Shi, L.; Liang, F.; Zheng, J.; Zhou, K.; Chen, S.; Yu, J.; Zhang, J. Melatonin regulates apoptosis and autophagy via ROS-MST1 pathway in subarachnoid hemorrhage. Front. Mol. Neurosci. 2018, 11, 93. [CrossRef] [PubMed]

24. Mortezaee, K.; Najafi, M.; Farhood, B.; Ahmadi, A.; Potes, Y.; Shabeeb, D.; Musa, A.E. Modulation of apoptosis by melatonin for improving cancer treatment efficiency: An updated review. Life Sci. 2019, 228, 228-241. [CrossRef] [PubMed]

25. Mills, E.; Wu, P.; Seely, D.; Guyatt, G. Melatonin in the treatment of cancer: A systematic review of randomized controlled trials and meta-analysis. J. Pineal Res. 2005, 39, 360-366. [CrossRef]

26. Zhao, M.; Wan, J.; Zeng, K.; Tong, M.; Lee, A.C.; Ding, J.; Chen, Q. The reduction in circulating melatonin level may contribute to the pathogenesis of ovarian cancer: A retrospective study. J. Cancer 2016, 7, 831-836. [CrossRef] [PubMed]

27. Garcia, C.P.; Lamarque, A.L.; Comba, A.; Berra, M.A.; Silva, R.A.; Labuckas, D.O.; Das, U.N.; Eynard, A.R.; Pasqualini, M.E. Synergistic anti-tumor effects of melatonin and PUFAs from walnuts in a murine mammary adenocarcinoma model. Nutrition 2015, 31, 570-577. [CrossRef] [PubMed]

28. Zhao, D.; Yu, Y.; Shen, Y.; Liu, Q.; Zhao, Z.; Sharma, R.; Reiter, R.J. Melatonin synthesis and function: Evolutionary history in animals and plants. Front. Endocrinol. 2019, 10, 249. [CrossRef] [PubMed]

29. Back, K.; Tan, D.-X.; Reiter, R.J. Melatonin biosynthesis in plants: Multiple pathways catalyze tryptophan to melatonin in the cytoplasm or chloroplasts. J. Pineal Res. 2016, 61, 426-437. [CrossRef] [PubMed]

30. Byeon, Y.; Choi, G.-H.; Lee, H.Y.; Back, K. Melatonin biosynthesis requires $N$-acetylserotonin methyltransferase activity of caffeic acid O-methyltransferase in rice. J. Exp. Bot. 2015, 66, 6917-6925. [CrossRef]

31. Byeon, Y.; Lee, H.J.; Lee, H.Y.; Back, K. Cloning and functional characterization of the Arabidopsis N-acetylserotonin O-methyltransferase responsible for melatonin synthesis. J. Pineal Res. 2016, 60, 65-73. [CrossRef] [PubMed]

32. Kang, K.; Lee, K.; Park, S.; Byeon, Y.; Back, K. Molecular cloning of rice serotonin $N$-acetyltransferase, the penultimate gene in plant melatonin biosynthesis. J. Pineal Res. 2013, 55, 7-13. [CrossRef] [PubMed]

33. Tan, D.X.; Hardeland, R.; Back, K.; Manchester, L.C.; Alatorre-Jimenez, M.A.; Reiter, R.J. On the significance of an alternate pathway of melatonin synthesis via 5-methoxytryptamine: Comparisons across species. J. Pineal Res. 2016, 61, 27-40. [CrossRef] [PubMed]

34. Lee, K.; Back, K. Overexpression of rice serotonin $N$-acetyltransferase 1 in transgenic rice plants confers resistance to cadmium and senescence and increases grain yield. J. Pineal Res. 2017, 62, e12392. [CrossRef] [PubMed]

35. Lee, H.Y.; Byeon, Y.; Lee, K.; Lee, H.J.; Back, K. Cloning of Arabidopsis serotonin N-acetyltransferase and its role with caffeic acid O-methyltransferase in the biosynthesis of melatonin in vitro despite their different subcellular localizations. J. Pineal Res. 2014, 57, 418-426. [CrossRef] [PubMed]

36. Byeon, Y.; Lee, H.Y.; Back, K. Cloning and characterization of the serotonin N-acetyltransferase-2 gene (SNAT2) in rice (Oryza sativa). J. Pineal Res. 2016, 61, 198-207. [CrossRef] [PubMed]

37. Pan, Q.H.; Chen, F.; Zhu, B.Q.; Ma, L.Y.; Li, L.; Li, J.M. Molecular cloning and expression of gene encoding aromatic amino acid decarboxylase in "Vidal blanc" grape berries. Mol. Biol. Rep. 2012, 39, 4319-4325. [CrossRef] [PubMed]

38. Yu, Y.; Bian, L.; Jiao, Z.; Yu, K.; Wan, Y.; Zhang, G.; Guo, D. Molecular cloning and characterization of a grapevine (Vitis vinifera L.) serotonin N-acetyltransferase (VvSNAT2) gene involved in plant defense. BMC Genom. 2019, 20, 880. [CrossRef]

39. Park, S.; Byeon, Y.; Lee, H.Y.; Kim, Y.-S.; Ahn, T.; Back, K. Cloning and characterization of a serotonin $N$-acetyltransferase from a gymnosperm, loblolly pine (Pinus taeda). J. Pineal Res. 2014, 57, 348-355. [CrossRef] [PubMed]

40. Sumi-Ichinose, C.; Ichinose, H.; Nagatsu, T.; Takahashi, E.I.; Hon, T.A. Molecular cloning of genomic DNA and chromosomal assignment of the gene for human aromatic L-Amino acid decarboxylase, the enzyme for catecholamine and serotonin biosynthesis. Biochemistry 1992, 31, 2229-2238. [CrossRef]

41. Kowlessur, D.; Kaufman, S. Cloning and expression of recombinant human pineal tryptophan hydroxylase in Escherichia coli: Purification and characterization of the cloned enzyme. Biochim. Biophys. Acta Protein Struct. Mol. Enzymol. 1999, 1434, $317-330$. [CrossRef]

42. Kim, K.S.; Wessel, T.C.; Stone, D.M.; Carver, C.H.; Joh, T.H.; Park, D.H. Molecular cloning and characterization of cDNA encoding tryptophan hydroxylase from rat central serotonergic neurons. Mol. Brain Res. 1991, 9, 277-283.

43. Taketoshi, M.; Horio, Y.; Imamura, I.; Tanaka, T.; Fukui, H.; Wada, H. Molecular cloning of guinea-pig aromatic-L-amino acid decarboxylase cDNA. Biochem. Biophys. Res. Commun. 1990, 170, 1229-1235. [CrossRef]

44. Florez, J.C.; Seidenman, K.J.; Barrett, R.K.; Sangoram, A.M.; Takahashi, J.S. Molecular cloning of chick pineal tryptophan hydroxylase and circadian oscillation of its mRNA levels. Mol. Brain Res. 1996, 42, 25-30. [CrossRef] 
45. Bellipanni, G.; Rink, E.; Bally-Cuif, L. Cloning of two tryptophan hydroxylase genes expressed in the diencephalon of the developing zebrafish brain. Mech. Dev. 2002, 119, S215-S220. [CrossRef]

46. Rahman, M.S.; Thomas, P. Molecular cloning, characterization and expression of two tryptophan hydroxylase (TPH-1 and TPH-2) genes in the hypothalamus of Atlantic croaker: Down-regulation after chronic exposure to hypoxia. Neuroscience 2009, 158, 751-765. [CrossRef]

47. Ma, T.; Tao, J.; Yang, M.; He, C.; Tian, X.; Zhang, X.; Zhang, J.; Deng, S.; Feng, J.; Zhang, Z.; et al. An AANAT/ASMT transgenic animal model constructed with CRISPR/Cas9 system serving as the mammary gland bioreactor to produce melatonin-enriched milk in sheep. J. Pineal Res. 2017, 63, e12406. [CrossRef]

48. Neckameyer, W.S.; White, K. A single locus encodes both phenylalanine hydroxylase and tryptophan hydroxylase activities in Drosophila. J. Biol. Chem. 1992, 267, 4199-4206. [CrossRef]

49. Coleman, C.M.; Neckameyer, W.S. Serotonin synthesis by two distinct enzymes in Drosophila melanogaster. Arch. Insect Biochem. Physiol. 2005, 59, 12-31. [CrossRef]

50. Hahn, S.L.; Hahn, M.; Joh, T.H. Genomic organization of the rat aromatic L-amino acid decarboxylase (AADC) locus: Partial analysis reveals divergence from the Drosophila dopa decarboxylase (DDC) gene structure. Mamm. Genome 1991, 1, 145-151. [CrossRef] [PubMed]

51. Hirsh, J.; Davidson, N. Isolation and characterization of the dopa decarboxylase gene of Drosophila melanogaster. Mol. Cell. Biol. 1981, 1, 475-485. [CrossRef] [PubMed]

52. Tatarenkov, A.; Ayala, F.J. Nucleotide variation at the dopa decarboxylase (Ddc) gene in natural populations of Drosophila melanogaster. J. Genet. 2007, 86, 125-137. [CrossRef] [PubMed]

53. Hintermann, E.; Jenö, P.; Meyer, U.A. Isolation and characterization of an arylalkylamine $N$-acetyltransferase from Drosophila melanogaster. FEBS Lett. 1995, 375, 148-150. [CrossRef]

54. Hintermann, E.; Grieder, N.C.; Amherd, R.; Brodbeck, D.; Meyer, U.A. Cloning of an arylalkylamine N-acetyltransferase (aaNAT1) from Drosophila melanogaster expressed in the nervous system and the gut. Proc. Natl. Acad. Sci. USA 1996, 93, 12315-12320. [CrossRef]

55. Amherd, R.; Hintermann, E.; Walz, D.; Affolter, M.; Meyer, U.A. Purification, cloning, and characterization of a second arylalkylamine N-Acetyltransferase from Drosophila melanogaster. DNA Cell Biol. 2000, 19, 697-705. [CrossRef]

56. Altschul, S.F.; Madden, T.L.; Schäffer, A.A.; Zhang, J.; Zhang, Z.; Miller, W.; Lipman, D.J. Gapped BLAST and PSI-BLAST: A new generation of protein database search programs. Nucleic Acids Res. 1997, 25, 3389-3402. [CrossRef]

57. Altschul, S.F.; Wootton, J.C.; Gertz, E.M.; Agarwala, R.; Morgulis, A.; Schaffer, A.A.; Yu, Y.-K. Protein database searches using compositionally adjusted substitution matrices. FEBS J. 2005, 272, 5101-5109. [CrossRef] [PubMed]

58. Flores-Gonzalez, M.; Hosmani, P.S.; Fernandez-Pozo, N.; Mann, M.; Humann, J.L.; Main, D.; Heck, M.; Brown, S.; Mueller, L.A.; Saha, S. Citrusgreening.org: An open access and integrated systems biology portal for the Huanglongbing (HLB) disease complex. bioRxiv 2019, 868364. [CrossRef]

59. Dempsey, D.R.; Jeffries, K.A.; Bond, J.D.; Carpenter, A.M.; Rodriguez-Ospina, S.; Breydo, L.; Caswell, K.K.; Merkler, D.J. Mechanistic and structural analysis of Drosophila melanogaster arylalkylamine N-acetyltransferases. Biochemistry 2014, 53, 7777-7793. [CrossRef] [PubMed]

60. Jones, D.T.; Taylor, W.R.; Thornton, J.M. The rapid generation of mutation data matrices from protein sequences. Bioinformatics 1992, 8, 275-282. [CrossRef]

61. Kumar, S.; Stecher, G.; Li, M.; Knyaz, C.; Tamura, K. MEGA X: Molecular evolutionary genetics analysis across computing platforms. Mol. Biol. Evol. 2018, 35, 1547-1549. [CrossRef] [PubMed]

62. Papadopoulos, J.S.; Agarwala, R. COBALT: Constraint-based alignment tool for multiple protein sequences. Bioinformatics 2007, 23, 1073-1079. [CrossRef] [PubMed]

63. Larkin, M.A.; Blackshields, G.; Brown, N.P.; Chenna, R.; McGettigan, P.A.; McWilliam, H.; Valentin, F.; Wallace, I.M.; Wilm, A.; Lopez, R.; et al. Clustal W and Clustal X version 2.0. Bioinformatics 2007, 23, 2947-2948. [CrossRef] [PubMed]

64. Blum, M.; Chang, H.Y.; Chuguransky, S.; Grego, T.; Kandasaamy, S.; Mitchell, A.; Nuka, G.; Paysan-Lafosse, T.; Qureshi, M.; Raj, S.; et al. The InterPro protein families and domains database: 20 years on. Nucleic Acids Res. 2021, 49, D344-D354. [CrossRef]

65. Bjellqvist, B.; Basse, B.; Olsen, E.; Celis, J.E. Reference points for comparisons of two-dimensional maps of proteins from different human cell types defined in a $\mathrm{pH}$ scale where isoelectric points correlate with polypeptide compositions. Electrophoresis 1994, 15, 529-539. [CrossRef] [PubMed]

66. Biasini, M.; Bienert, S.; Waterhouse, A.; Arnold, K.; Studer, G.; Schmidt, T.; Kiefer, F.; Cassarino, T.G.; Bertoni, M.; Bordoli, L.; et al. SWISS-MODEL: Modelling protein tertiary and quaternary structure using evolutionary information. Nucleic Acids Res. 2014, 42, W252-W258. [CrossRef] [PubMed]

67. Kelley, L.A.; Mezulis, S.; Yates, C.M.; Wass, M.N.; Sternberg, M.J.E. The Phyre2 web portal for protein modeling, prediction and analysis. Nat. Protoc. 2015, 10, 845-858. [CrossRef]

68. Pettersen, E.F.; Goddard, T.D.; Huang, C.C.; Couch, G.S.; Greenblatt, D.M.; Meng, E.C.; Ferrin, T.E. UCSF Chimera? A visualization system for exploratory research and analysis. J. Comput. Chem. 2004, 25, 1605-1612. [CrossRef]

69. Lorenz, R.; Bernhart, S.H.; Höner zu Siederdissen, C.; Tafer, H.; Flamm, C.; Stadler, P.F.; Hofacker, I.L. ViennaRNA Package 2.0. Algorithms Mol. Biol. 2011, 6, 26. [CrossRef] 
70. Tatineni, S.; Sagaram, U.S.; Gowda, S.; Robertson, C.J.; Dawson, W.O.; Iwanami, T.; Wang, N. In planta distribution of “Candidatus Liberibacter asiaticus" as revealed by polymerase chain reaction (PCR) and real-time PCR. Phytopathology 2008, 98, 592-599. [CrossRef]

71. Livak, K.J.; Schmittgen, T.D. Analysis of relative gene expression data using real-time quantitative PCR and the $2^{-\Delta \Delta C}$ T Method. Methods 2001, 25, 402-408. [CrossRef] [PubMed]

72. Murch, S.J.; KrishnaRaj, S.; Saxena, P.K. Tryptophan is a precursor for melatonin and serotonin biosynthesis in in vitro regenerated St. John's wort (Hypericum perforatum L. cv. Anthos) plants. Plant Cell Rep. 2000, 19, 698-704. [CrossRef] [PubMed]

73. Duan, Y.; Zhou, L.; Hall, D.G.; Li, W.; Doddapaneni, H.; Lin, H.; Liu, L.; Vahling, C.M.; Gabriel, D.W.; Williams, K.P.; et al. Complete genome sequence of citrus huanglongbing bacterium, “Candidatus Liberibacter asiaticus" obtained through metagenomics. Mol. Plant. Microbe Interact. 2009, 22, 1011-1020. [CrossRef] [PubMed]

74. Wang, L.; Erlandsen, H.; Haavik, J.; Knappskog, P.M.; Stevens, R.C. Three-dimensional structure of human tryptophan hydroxylase and its implications for the biosynthesis of the neurotransmitters serotonin and melatonin. Biochemistry 2002, 41, 12569-12574. [CrossRef] [PubMed]

75. Grenett, H.E.; Ledley, F.D.; Reed, L.L.; Woo, S.L. Full-length cDNA for rabbit tryptophan hydroxylase: Functional domains and evolution of aromatic amino acid hydroxylases. Proc. Natl. Acad. Sci. USA 1987, 84, 5530-5534. [CrossRef]

76. Hoang, L.; Byck, S.; Prevost, L.; Scriver, C.R. PAH Mutation Analysis Consortium Database: A database for disease-producing and other allelic variation at the human PAH locus. Nucleic Acids Res. 1996, 24, 125-126. [CrossRef]

77. Erlandsen, H.; Hough, E.; Fusetti, F.; Stevens, R.C.; Fusetti, F.; Stevens, R.C.; Martinez, A.; Flatmark, T.; Fusetti, F. Crystal structure of the catalytic domain of human phenylalanine hydroxylase reveals the structural basis for phenylketonuria. Nat. Struct. Biol. 1997, 4, 995-1000. [CrossRef] [PubMed]

78. Pereira, G.R.C.; Tavares, G.D.B.; de Freitas, M.C.; De Mesquita, J.F. In silico analysis of the tryptophan hydroxylase 2 (TPH2) protein variants related to psychiatric disorders. PLoS ONE 2020, 15, e0229730. [CrossRef] [PubMed]

79. Goodwill, K.E.; Sabatier, C.; Marks, C.; Raag, R.; Fitzpatrick, P.F.; Stevens, R.C. Crystal structure of tyrosine hydroxylase at $2.3 \AA$ and its implications for inherited neurodegenerative diseases. Nat. Struct. Biol. 1997, 4, 578-585. [CrossRef] [PubMed]

80. Sandmeier, E.; Hale, T.I.; Christen, P. Multiple evolutionary origin of pyridoxal-5'-phosphate-dependent amino acid decarboxylases. Eur. J. Biochem. 1994, 221, 997-1002. [CrossRef] [PubMed]

81. Ishii, S.; Mizuguchi, H.; Nishino, J.; Hayashi, H.; Kagamiyama, H. Functionally important residues of aromatic L-amino acid decarboxylase probed by sequence alignment and site-directed mutagenesis. J. Biochem. 1996, 120, 369-376. [CrossRef] [PubMed]

82. Jackson, F.R. Prokaryotic and eukaryotic pyridoxal-dependent decarboxylases are homologous. J. Mol. Evol. 1990, 31, 325-329. [CrossRef] [PubMed]

83. Joseph, D.R.; Sullivan, P.M.; Wang, Y.M.; Kozak, C.; Fenstermacher, D.A.; Behrendsen, M.E.; Zahnow, C.A. Characterization and expression of the complementary DNA encoding rat histidine decarboxylase. Proc. Natl. Acad. Sci. USA 1990, 87, 733-737. [CrossRef] [PubMed]

84. Napolitano, L.; Galluccio, M.; Scalise, M.; Parravicini, C.; Palazzolo, L.; Eberini, I.; Indiveri, C. Novel insights into the transport mechanism of the human amino acid transporter LAT1 (SLC7A5). Probing critical residues for substrate translocation. Biochim. Biophys. Acta Gen. Subj. 2017, 1861, 727-736. [CrossRef] [PubMed]

85. Brodbeck, D.; Amherd, R.; Callaerts, P.; Hintermann, E.; Meyer, U.A.; Affolter, M. Molecular and biochemical characterization of the aaNAT1 (Dat) locus in Drosophila melanogaster: Differential expression of two gene products. DNA Cell Biol. 1998, 17, 621-633. [CrossRef] [PubMed]

86. Klein, D.C. Arylalkylamine N-acetyltransferase: “The timezyme". J. Biol. Chem. 2007, 282, 4233-4237. [CrossRef]

87. Axelrod, J.; Weissbach, H. Purification and properties of hydroxyindole-O-methyl transferase. J. Biol. Chem. 1961, 236, 211-213. [CrossRef]

88. Liu, T.; Borjigin, J. N-acetyltransferase is not the rate-limiting enzyme of melatonin synthesis at night. J. Pineal Res. 2005, 39, 91-96. [CrossRef] [PubMed] 